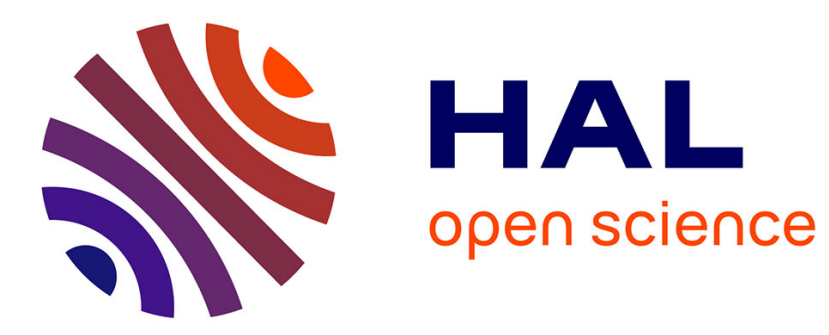

\title{
Identification of the effects of the nozzle-exit boundary-layer thickness and its corresponding Reynolds number in initially highly disturbed subsonic jets
}

Christophe Bogey, Olivier Marsden

\section{- To cite this version: \\ Christophe Bogey, Olivier Marsden. Identification of the effects of the nozzle-exit boundary-layer thickness and its corresponding Reynolds number in initially highly disturbed subsonic jets. Physics of Fluids, 2013, 25, pp.055106. 10.1063/1.4807071 · hal-00931418}

\author{
HAL Id: hal-00931418 \\ https://hal.science/hal-00931418
}

Submitted on 8 Apr 2016

HAL is a multi-disciplinary open access archive for the deposit and dissemination of scientific research documents, whether they are published or not. The documents may come from teaching and research institutions in France or abroad, or from public or private research centers.
L'archive ouverte pluridisciplinaire $\mathbf{H A L}$, est destinée au dépôt et à la diffusion de documents scientifiques de niveau recherche, publiés ou non, émanant des établissements d'enseignement et de recherche français ou étrangers, des laboratoires publics ou privés. 


\title{
Identification of the effects of the nozzle-exit boundary-layer thickness and its corresponding Reynolds number in initially highly disturbed subsonic jets
}

\author{
Christophe Bogey ${ }^{\text {a) }}$ and Olivier Marsden \\ Laboratoire de Mécanique des Fluides et d'Acoustique, UMR CNRS 5509, \\ Ecole Centrale de Lyon, Université de Lyon, 69134 Ecully Cedex, France
}

(Received 17 December 2012; accepted 24 April 2013; published online 31 May 2013)

\begin{abstract}
The influence of the nozzle-exit boundary-layer thickness in isothermal round jets at a Mach number of 0.9 and at diameter Reynolds numbers $\operatorname{Re}_{D} \simeq 5 \times 10^{4}$ is investigated using large-eddy simulations. The originality of this work is that, contrary to previous studies on the topic, the jets are initially highly disturbed, and that the effects of the boundary-layer thickness are explored jointly on the exit turbulence, the shear-layer and jet flow characteristics, and the acoustic field. The jets originate from a pipe of radius $r_{0}$, and exhibit, at the exit, peak disturbance levels of $9 \%$ of the jet velocity, and mean velocity profiles similar to laminar boundary-layer profiles of thickness $\delta_{0}=0.09 r_{0}, 0.15 r_{0}, 0.25 r_{0}$, or $0.42 r_{0}$, yielding $99 \%$ velocity thicknesses between $0.07 r_{0}$ and $0.34 r_{0}$ and momentum thicknesses $\delta_{\theta}(0)$ between $0.012 r_{0}$ and $0.05 r_{0}$. Two sets of computations are reported to distinguish, for the first time to the best of our knowledge, between the effects of the ratio $\delta_{0} / r_{0}$ and of the Reynolds number $\operatorname{Re}_{\theta}$ based on $\delta_{\theta}(0)$. First, four jets with a fixed diameter, hence at a constant Reynolds number $\operatorname{Re}_{D}=5 \times 10^{4}$ giving $\operatorname{Re}_{\theta}=304,486,782$, and 1288 depending on $\delta_{0}$, are considered. In this case, due to the increase in $\operatorname{Re}_{\theta}$, thickening the initial shear layers mainly results in a weaker mixing-layer development with lower spreading rates and turbulence intensities, and reduced sound levels at all emission angles. Second, four jets at Reynolds numbers $\operatorname{Re}_{D}$ between $1.8 \times 10^{4}$ and $8.3 \times 10^{4}$, varying so as to obtain $\operatorname{Re}_{\theta} \simeq 480$ in all simulations, are examined. Here, increasing $\delta_{0} / r_{0}$ has a limited impact on the mixing-layer key features, but clearly leads to a shorter potential core, a more rapid velocity decay, and higher fluctuations on the jet axis, and stronger noise in the downstream direction. Similar trends can be expected for high-Reynolds-number jets in which viscosity plays a negligible role. (C) 2013 AIP Publishing LLC. [http://dx.doi.org/10.1063/1.4807071]
\end{abstract}

\section{INTRODUCTION}

The crucial role of the initial conditions in free shear flows has been well recognized for more than thirty years. The turbulent development and acoustic field of jets have in particular been shown to depend on nozzle-exit parameters such as the Reynolds number, the mean velocity profile, the thickness and the shape factor of the boundary layer, and the disturbance level, just to mention a few important ones, as pointed out in the reference papers by Hussain ${ }^{1}$ and Crighton. ${ }^{2}$ These parameters are likely to vary significantly from one experiment to another. This is notably the case for the initial shear-layer thickness $\delta_{0}$ or momentum thickness $\delta_{\theta}(0)$. In two recent experimental studies on round jets of diameter $D$ or radius $r_{0}$, for example, Morris and Zaman ${ }^{3}$ measured $\delta_{\theta}(0)$ $=0.0013 r_{0}$ in a jet at a Mach number $\mathrm{M}=0.25$ and at a diameter-based Reynolds number $\operatorname{Re}_{D}=3$ $\times 10^{5}$, whereas Arakeri et al. ${ }^{4}$ found $\delta_{\theta}(0) \simeq 0.04 r_{0}$ in a jet at $\mathrm{M}=0.9$ and $\mathrm{Re}_{D}=5 \times 10^{5}$.

\footnotetext{
a)Electronic mail: christophe.bogey@ec-lyon.fr
} 
The thickness of the nozzle-exit boundary layer in jets, however, appears to follow general trends depending on its laminar, transitional or turbulent state. In initially laminar jets, it first decreases with increasing Reynolds number following a $1 / \operatorname{Re}_{D}^{1 / 2}$ proportionality law, which was predicted, for instance, by Becker and Massaro ${ }^{10}$ based on simple boundary-layer concepts, and was verified experimentally by Crow and Champagne,${ }^{5}$ Zaman, ${ }^{6-8}$ and Deo et al.${ }^{9}$ In this case, the constant of proportionality setting the boundary-layer thickness obviously depends on the nozzle geometry and contraction ratio. The influence of a constant-diameter tail-pipe attached to the downstream end of the nozzle was, for example, explored by Ahuja ${ }^{11,12}$ and Hasan and Hussain. ${ }^{13}$ Tests were also recently performed using three nozzles of identical exit diameter, differing only in internal profile. The results obtained using the so-called ASME, cubic and conic nozzles have been discussed by Viswanathan and Clark, ${ }^{14}$ Harper-Bourne, ${ }^{15}$ Zaman, ${ }^{8}$ and Karon and Ahuja. ${ }^{12}$ When the nozzle-exit flow conditions become turbulent, the boundary-layer thickness then sharply rises by a factor of between 2 and 4 with respect to the laminar case. This jump is observed when the laminar-turbulent transition in the nozzle is forced by a tripping device as in Zaman, ${ }^{6,7}$ Bridges and Hussain, ${ }^{16}$ Raman et al. ${ }^{17}$ or Morris and Zaman, ${ }^{3}$ as well as when it occurs naturally as in Zaman. ${ }^{8}$ In initially turbulent jets, according to the latter author, ${ }^{8}$ the nozzle-exit boundary-layer thickness finally decreases only marginally with the Reynolds number.

The effects of the initial shear-layer thickness in jets are unfortunately difficult to investigate experimentally, because it cannot usually be modified independently of the initial turbulence level. Furthermore, experiments are generally performed for jets with a fixed diameter, hence at a constant Reynolds number $\operatorname{Re}_{D}$ but at varying momentum-thickness-based Reynolds numbers $\operatorname{Re}_{\theta}$ when the value of $\delta_{0}$ is changed. This was the case in the work by Hussain and Zedan, ${ }^{18}$ which dealt with axisymmetric mixing layers characterized initially by similar peak rms velocities $u_{e}^{\prime} / u_{j} \simeq 6 \%$ and different thicknesses yielding Reynolds numbers $\operatorname{Re}_{\theta}$ between 184 and 349. This remark also applies to the recent jet experiments using ASME and conic nozzles. For jets at $\mathrm{M}=0.37$ and $\operatorname{Re}_{D}=2.2$ $\times 10^{5}$, Zaman ${ }^{8}$ found, for instance, exit-condition parameters equal to $\delta_{\theta}=0.0053 r_{0}$ and $\operatorname{Re}_{\theta}$ $=1180$ with the ASME nozzle, and to $\delta_{\theta}=0.0025 r_{0}$ and $\operatorname{Re}_{\theta}=560$ with the conic nozzle. For jets at $\mathrm{M}=0.4$ and $\operatorname{Re}_{D}=3.5 \times 10^{5}$, Karon and Ahuja ${ }^{12}$ similarly measured $\delta_{\theta}=0.0032 r_{0}$ and $\operatorname{Re}_{\theta}$ $=1130$, and $\delta_{\theta}=0.0024 r_{0}$ and $\operatorname{Re}_{\theta}=870$, respectively. The variations with the initial shear-layer thickness $\delta_{0}$ obtained in this way can be expected to result from the modification of both the ratio $\delta_{0} / r_{0}$ and the value of $\operatorname{Re}_{\theta}$, or, in other words, from both geometry and viscosity effects.

The influence of the two parameters above on linear instability waves has been investigated theoretically by Michalke ${ }^{19}$ and Cohen and Wygnanski ${ }^{20}$ for the former, and by Morris ${ }^{21,22}$ for the latter. For hyperbolic-tangent axisymmetric velocity profiles, it was found that the amplification curves of the first azimuthal disturbances vary significantly with the ratio $\delta_{\theta} / r_{0}$ for $\delta_{\theta} / r_{0} \geq 0.08$, and with the Reynolds number for $\operatorname{Re}_{\theta} \leq 80$. For $\delta_{\theta} / r_{0}<0.08$ and $\operatorname{Re}_{\theta}>80$, the changes are less marked, and increasing $\delta_{\theta} / r_{0}$ or $\operatorname{Re}_{\theta}$ generally leads to lower growth rates, but does not fundamentally alter the Strouhal number $\mathrm{St}_{\theta}$ of maximum amplification, in agreement with the measurements collected in Gutmark and Ho. ${ }^{23}$ Finally, the dependence on $\delta_{\theta} / r_{0}$ and $\operatorname{Re}_{\theta}$ is quite small for $\delta_{\theta} / r_{0} \leq 0.04$ and for $\operatorname{Re}_{\theta} \geq 320$, that is to say for the values of shear-layer thickness and Reynolds number encountered in most experiments. These results are of clear interest for initially laminar jets, but are less applicable to initially turbulent jets, for which the modeling of velocity fluctuations by instability waves is not straightforward, refer to the work by Gudmundsson and Colonius. ${ }^{24}$ Based on a spatial stability analysis, it appears difficult to predict, for example, the effects of the Reynolds number, which are known to be important in turbulent mixing layers. As $\mathrm{Re}_{\theta}$ increases, more fine-scale structures are in particular observed, as shown by the experiments of Brown and Roshko, ${ }^{25}$ or by the simulations of Kleinman and Freund. ${ }^{26}$ The shear layers also develop more slowly, with lower levels of velocity fluctuations, according to the calculations of Bogey et al. ${ }^{27}$

Given the issues outlined previously, and the rapid progress in jet computation reviewed, for instance, in the papers by Colonius and Lele, ${ }^{28}$ Bailly and Bogey ${ }^{29}$ and Wang et al., ${ }^{30}$ it appears interesting to perform simulations in order to carefully investigate the effects of the initial shearlayer thickness on jets. It can be noted that a number of numerical studies have already been conducted on this matter over the last thirteen years. Stanley and Sarkar ${ }^{31}$ and Bogey and Bailly ${ }^{32}$ first examined plane and round jets without including a nozzle in the computational domain, for which 
hyperbolic-tangent velocity profiles were imposed at the inflow boundary. Kim and $\mathrm{Choi}^{33}$ and Bogey and Bailly ${ }^{34}$ later focused on round jets with fully laminar conditions downstream of a pipe nozzle. In the work of the latter authors, jets at a Mach number of 0.9 and at a Reynolds number $\operatorname{Re}_{D}=$ $10^{5}$, with an inlet boundary-layer thickness $\delta_{0}=0.025,0.05,0.1$, and 0.2 , were considered. Shorter potential cores and higher centreline turbulence intensities were observed with increasing $\delta_{0}$. In all cases, however, the shear-layer transitions were dominated by vortex rolling-ups and pairings, generating strong noise components, which is less prone to happen in initially turbulent jets.

To extend and complete the previous studies reported above, the present work aims to carefully investigate the influence of the nozzle-exit boundary-layer thickness in initially highly disturbed subsonic round jets, jointly on the initial turbulence, the shear-layer and jet flow developments, and the acoustic field. Moreover, the effects of both the boundary-layer thickness and its corresponding Reynolds number are considered, for the first time to the best of our knowledge. Large-eddy simulations (LES) based on low-dissipation schemes and relaxation filtering as subgrid model are carried out. The jets are at a Mach number of 0.9 , and at moderate Reynolds numbers $\operatorname{Re}_{D}$ between 1.8 $\times 10^{4}$ and $8.3 \times 10^{4}$ to ensure the numerical accuracy of the LES using a $252 \times 10^{6}$ point grid. ${ }^{35,36}$ In the same way as in recent studies, devoted notably to the importance of the initial disturbance level ${ }^{37}$ and of the Reynolds number, ${ }^{27}$ a trip-like excitation is applied to the jet boundary layers in a pipe nozzle, in order to obtain, at the exit, peak turbulence intensities $u_{e}^{\prime} / u_{j}=9 \%$, where $u_{j}$ is the jet velocity, and mean velocity profiles similar to Blasius laminar profiles. Seven jet simulations are reported here. The first jet has a Reynolds number $\operatorname{Re}_{D}=5 \times 10^{4}$ and a boundary-layer thickness $\delta_{0}$ $=0.15 r_{0}$, yielding $\delta_{\theta}(0)=0.019 r_{0}$ and $\operatorname{Re}_{\theta}=486$ at the nozzle exit. ${ }^{27}$ The next three jets are also at $\operatorname{Re}_{D}=5 \times 10^{4}$, but have either thinner or thicker initial shear layers with respect to the first case. Values $\delta_{0}=0.09 r_{0}, 0.25 r_{0}$, and $0.42 r_{0}$ are chosen, leading to $\operatorname{Re}_{\theta}$ around 300,800 , and 1300 . The same ratios $\delta_{0} / r_{0}=0.09,0.25$, and 0.42 are specified in the last three jets. The diameter Reynolds numbers are, however, set to $\mathrm{Re}_{D}=8.3 \times 10^{4}, 3 \times 10^{4}$, and $1.8 \times 10^{4}$, respectively, in order to maintain $\operatorname{Re}_{\theta} \simeq 480$ in all cases. In this way, we will be able to distinguish between the effects of $\delta_{0} / r_{0}$ and $\operatorname{Re}_{\theta}$ on the jet characteristics.

The paper is organized as follows. In Sec. II, the main parameters of the jets and of the simulations are documented. In Sec. III, the nozzle-exit flow conditions, and the aerodynamic and acoustic fields obtained for the four jets at $\operatorname{Re}_{D}=5 \times 10^{4}$ are first presented. The results for the jets at $\operatorname{Re}_{\theta} \simeq 480$ are then shown. Concluding remarks are provided in Sec. IV. The amplification rates of linear instability waves in mixing layers representative of the mean flows obtained downstream of the jet nozzle exit are finally examined in the Appendix.

\section{PARAMETERS}

In this section, the jet inflow conditions are first presented. The numerical methods and parameters are then briefly reported. They are identical to those used in recent jet simulations, which have been thoroughly described in previous references. ${ }^{27,34-37}$ The simulation of the jet at $\operatorname{Re}_{D}=5 \times 10^{4}$ with a boundary-layer thickness $\delta_{0}=0.15 r_{0}$ considered in the present study was moreover detailed in Bogey et al. ${ }^{27}$ A great amount of information about the boundary-layer tripping procedure, the discretization quality, and the LES reliability is also available in papers ${ }^{35,36}$ dealing with jets at $\operatorname{Re}_{D}=10^{5}$.

\section{A. Jet definition}

Seven isothermal round jets at a Mach number $\mathrm{M}=u_{j} / c_{a}=0.9$ and at Reynolds numbers $\operatorname{Re}_{D}$ $=u_{j} D / v$ over the range $1.8 \times 10^{4} \leq \operatorname{Re}_{D} \leq 8.3 \times 10^{4}$, where $c_{a}$ is the ambient speed of sound and $v$ is the kinematic molecular viscosity, are investigated. They originate at $z=0$ from a pipe nozzle of radius $r_{0}$ and length $2 r_{0}$, whose lip is $0.053 r_{0}$ thick, as represented in Figure 1(a). The ambient temperature and pressure are $T_{a}=293 \mathrm{~K}$ and $p_{a}=10^{5} \mathrm{~Pa}$. For all jets, the axial velocity profile at the pipe inlet is given by an approximated solution of the Blasius laminar boundary-layer profile. More precisely, a Pohlhausen's fourth-order polynomial profile of thickness $\delta_{0}$, yielding a $99 \%$ velocity thickness $\delta_{99}=0.82 \delta_{0}$, is imposed. ${ }^{34}$ Radial and azimuthal velocities are initially set to zero, pressure 
(a)

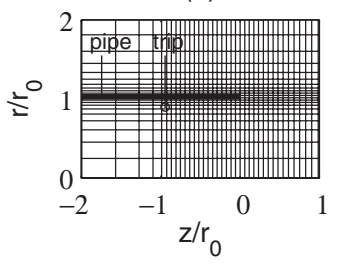

(b)

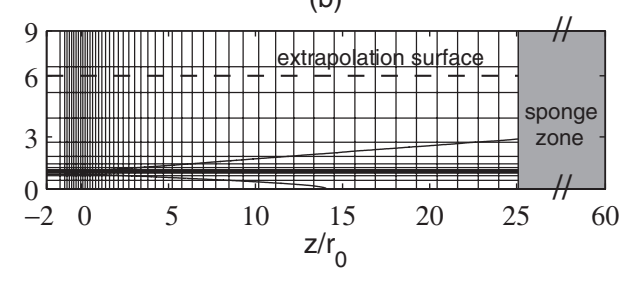

FIG. 1. Visualization in the $(z, r)$ plane of the LES mesh grid: (a) pipe region and (b) full computational domain; every 8th and 18th grid points are plotted, respectively. In (b), the contours obtained for the jet at $\operatorname{Re}_{D}=5 \times 10^{4}$ with $\delta_{0}=0.25 r_{0}$ for mean axial velocities of $0.95 u_{j}$ and $0.1 u_{j}$ are represented. Only $r \geq 0$ is shown.

is set to $p_{a}$, and the temperature is determined by a Crocco-Busemann relation. In order to generate highly disturbed upstream conditions for the jets, whose initial state would otherwise be laminar, a trip-like forcing is applied to the boundary layers at $z=-0.95 r_{0}$ inside the pipe by adding random low-level vortical disturbances decorrelated in the azimuthal direction. The excitation magnitudes are empirically chosen to obtain, at the pipe exit, peak turbulence intensities $u_{e}^{\prime} / u_{j}$ around $9 \%$ as in the tripped subsonic jets of Zaman, ${ }^{6,7}$ and mean velocity profiles remaining similar to the Blasius laminar profiles introduced at the pipe inlet, which will be illustrated in Secs. III A 1 and III B 1. Pressure fluctuations of maximum amplitude $200 \mathrm{~Pa}$ random in both space and time are also added in the shear layers between $z=0.25 r_{0}$ and $z=4 r_{0}$ from $t=0$ up to non-dimensional time $t=12.5 r_{0} / u_{j}$, in order to speed up the initial transitory period.

As shown in Table I, four boundary-layer thicknesses $\delta_{0}=0.09 r_{0}, 0.15 r_{0}, 0.25 r_{0}$, and $0.42 r_{0}$, corresponding to $99 \%$ velocity thicknesses $\delta_{99}=0.07 r_{0}, 0.12 r_{0}, 0.20 r_{0}$, and $0.34 r_{0}$, respectively, are considered at the pipe inlet. They will result in nozzle-exit momentum thicknesses over the range $0.012 r_{0} \leq \delta_{\theta}(0) \leq 0.05 r_{0}$. The initial shear layers of the jets are therefore relatively thick. This situation is encountered in some experiments on tripped jets, such as those of Zaman ${ }^{6,7}$ and Arakeri et al. ${ }^{4}$ in which $\delta_{\theta}(0)=0.018 r_{0}$ and $\delta_{\theta}(0) \simeq 0.04 r_{0}$ were measured at $\operatorname{Re}_{D} \simeq 10^{5}$ and $\operatorname{Re}_{D}=5$ $\times 10^{5}$, respectively. In a first step, jets at a constant $\operatorname{Re}_{D}=5 \times 10^{4}$, hence at momentum-thickness Reynolds numbers $\operatorname{Re}_{\theta}=u_{j} \delta_{\theta}(0) / v$ between about 300 and 1300 depending on $\delta_{0} / r_{0}$, are examined, refer to Table I(a). In a second step, conversely, the diameter Reynolds number of the jets varies in such a way that $\operatorname{Re}_{\theta} \simeq 480$ is achieved in all cases, as indicated in Table $\mathrm{I}(\mathrm{b})$.

For completeness, the amplitude of the trip-like excitation applied to the jet boundary layers to reach $u_{e}^{\prime} / u_{j} \simeq 9 \%$ is provided. The coefficient $\alpha$ specifying the forcing strength, refer to Appendix A in Bogey et al., ${ }^{35}$ is set to values of $0.091,0.068,0.0625$, and 0.07075 for the jets at

TABLE I. Inflow conditions for the jets (a) at $\operatorname{Re}_{D}=5 \times 10^{4}$ and (b) at $\operatorname{Re}_{\theta} \simeq 480$ : Mach and Reynolds numbers $M$ and $\operatorname{Re}_{D}$, inlet boundary-layer thickness $\delta_{0}$, and intended Reynolds number $\operatorname{Re}_{\theta}$ based on the nozzle-exit boundary-layer momentum thickness.

\begin{tabular}{|c|c|c|c|}
\hline M & $\operatorname{Re}_{D}$ & $\delta_{0} / r_{0}$ & $\operatorname{Re}_{\theta}$ \\
\hline \multicolumn{4}{|c|}{ (a) $\operatorname{Re}_{D}=5 \times 10^{4}$} \\
\hline 0.9 & $5 \times 10^{4}$ & 0.09 & $\sim 300$ \\
\hline 0.9 & $5 \times 10^{4}$ & 0.15 & $\sim 480$ \\
\hline 0.9 & $5 \times 10^{4}$ & 0.25 & $\sim 800$ \\
\hline 0.9 & $5 \times 10^{4}$ & 0.42 & $\sim 1300$ \\
\hline \multicolumn{4}{|c|}{ (b) $\operatorname{Re}_{\theta} \simeq 480$} \\
\hline 0.9 & $8.3 \times 10^{4}$ & 0.09 & $\sim 480$ \\
\hline 0.9 & $5 \times 10^{4}$ & 0.15 & $\sim 480$ \\
\hline 0.9 & $3 \times 10^{4}$ & 0.25 & $\sim 480$ \\
\hline 0.9 & $1.8 \times 10^{4}$ & 0.42 & $\sim 480$ \\
\hline
\end{tabular}


TABLE II. Simulation parameters: numbers of grid points $n_{r}, n_{\theta}$ and $n_{z}$, mesh spacings $\Delta r$ at $r=r_{0}, r_{0} \Delta \theta$, and $\Delta z$ at $z=$ 0 , extents $L_{r}$ and $L_{z}$ of the physical domain, radial position $r_{c}$ of the far-field extrapolation surface, and time duration $T$.

\begin{tabular}{lcccccc}
\hline \hline$n_{r}, n_{\theta}, n_{z}$ & $\Delta r / r_{0}$ & $r_{0} \Delta \theta / r_{0}$ & $\Delta z / r_{0}$ & $L_{r}, L_{z}$ & $r_{c} / r_{0}$ & $T u_{j} / r_{0}$ \\
\hline $256,1024,962$ & $0.36 \%$ & $0.61 \%$ & $0.72 \%$ & $9 r_{0}, 25 r_{0}$ & 6.5 & 375 \\
\hline \hline
\end{tabular}

$\operatorname{Re}_{D}=5 \times 10^{4}$ with $\delta_{0}=0.09 r_{0}, 0.15 r_{0}, 0.25 r_{0}$, and $0.42 r_{0}$, respectively. It is equal to 0.05575 , $0.068,0.0845$, and 0.01125 for the jets at $\operatorname{Re}_{\theta} \simeq 480$ with increasing $\delta_{0} / r_{0}$. Thus, in the latter case, the thicker the boundary layers, the higher the magnitude of the forcing necessary to reach a given level of nozzle-exit velocity disturbances, as expected.

\section{B. LES procedure and numerical methods}

The LES are carried out using a solver of the three-dimensional filtered compressible NavierStokes equations in cylindrical coordinates $(r, \theta, z)$ based on low-dissipation and low-dispersion explicit schemes. The axis singularity is taken into account by the method of Mohseni and Colonius. ${ }^{38}$ To alleviate the time-step restriction near the cylindrical origin, the derivatives in the azimuthal direction around the axis are calculated at coarser resolutions than permitted by the grid. ${ }^{39}$ Fourthorder eleven-point centered finite differences are used for spatial discretization, and a second-order six-stage Runge-Kutta algorithm is implemented for time integration. ${ }^{40} \mathrm{~A}$ sixth-order eleven-point centered filter ${ }^{41}$ is applied explicitly to the flow variables every time step. Non-centered finite differences and filters are also used near the pipe walls and the grid boundaries..$^{34,42}$ The radiation conditions of Tam and Dong ${ }^{43}$ are applied at all boundaries, with the addition at the outflow of a sponge zone combining grid stretching and Laplacian filtering. ${ }^{44}$

In the simulations, the explicit filtering is employed to remove grid-to-grid oscillations, but also as a subgrid high-order dissipation model to relax turbulent energy from scales at wave numbers close to the grid cut-off wave number while leaving larger scales mostly unaffected. ${ }^{45-48}$ With this in mind, the reliability of the LES fields obtained for a jet at $\operatorname{Re}_{D}=10^{5}$ with $u_{e}^{\prime} / u_{j}=9 \%$ and $\delta_{0}=0.15 r_{0}$ using the same grid as in this study has been assessed in Bogey et al. ${ }^{35}$ based on the transfer functions associated with molecular viscosity, relaxation filtering, and time integration. Viscosity was shown to be the dominant dissipation mechanism for scales discretized at least by seven points per wavelength. The physics of the larger turbulent structures is therefore unlikely to be governed by numerical or subgrid-modeling dissipation, implying in particular that the effective flow Reynolds number should not be artificially decreased. These remarks certainly equally hold for the present simulations dealing with jets at lower Reynolds numbers.

\section{Simulation parameters}

The computational domain is illustrated in Figure 1. As reported in Table II, the LES are performed using a grid containing $n_{r} \times n_{\theta} \times n_{z}=256 \times 1024 \times 962=252 \times 10^{6}$ points. There are 169 points along the pipe nozzle, 77 points within the jet radius, and 23, 31, 42, or 53 points inside the inlet boundary layers depending on the ratio $\delta_{0} / r_{0}$. The physical domain, excluding the eighty-point outflow sponge zone, extends axially down to $L_{z}=25 r_{0}$, and radially out to $L_{r}=9 r_{0}$.

The mesh spacing is uniform in the azimuthal direction, with $r_{0} \Delta \theta=0.0061 r_{0}$. In the axial direction, the mesh spacing is minimum between $z=-r_{0}$ and $z=0$, with $\Delta z=0.0072 r_{0}$. It increases upstream of $z=-r_{0}$, but also downstream of the nozzle at stretching rates lower than $1 \%$ allowing to reach $\Delta z=0.065 r_{0}$ between $z=13.3 r_{0}$ and $z=L_{z}=25 r_{0}$. In the radial direction, the mesh spacing is minimum around $r=r_{0}$, with $\Delta r=0.0036 r_{0}$. It is equal to $\Delta r=0.292 r_{0}$ close to the jet axis, to $\Delta r=0.081 r_{0}$ between $r=3 r_{0}$ and $r=6.75 r_{0}$, and finally to $\Delta r=0.176 r_{0}$ at $r=L_{r}=9 r_{0}$. Further details regarding the mesh spacings can be found in previous papers. ${ }^{35,37}$ 
The grid quality has been discussed in Bogey et al. ${ }^{35}$ for a jet at $\operatorname{Re}_{D}=10^{5}$ with $u_{e}^{\prime} / u_{j}=9 \%$, $\delta_{0}=0.15 r_{0}$, and $\operatorname{Re}_{\theta}=900$. The ratios between the integral length scales of the axial fluctuating velocity and the mesh spacings along the lip line were shown to fall between 4 and 10 . The properties of the nozzle-exit turbulence and of the shear-layer flow fields were moreover found to be practically converged with respect to the grid. Based on these results, there seems little doubt that in this study the grid resolution is appropriate for the jets with $\delta_{0}=0.15 r_{0}, 0.25 r_{0}$, or $0.42 r_{0}$. Indeed, they display as thick or thicker mixing layers and lower diameter-based Reynolds numbers than the jet mentioned above, both of which points argue in favour of higher numerical accuracy. The resolution is also probably sufficient for the two jets with $\delta_{0}=0.09 r_{0}$, that is, with thinner initial shear layers, because of the momentum-thickness Reynolds numbers $\operatorname{Re}_{\theta} \simeq 300$ and 480 which are much lower than that of $\operatorname{Re}_{\theta} \simeq 900$ in the reference jet. This should lead, for instance, to an increase by at least $25 \%$ of the integral length scales according to a recent work. ${ }^{27}$ Note that the mixing-layer flow fields obtained for the four jets at $\operatorname{Re}_{\theta} \simeq 480$ will later confirm the good grid quality in the present LES.

The simulation time, given in Table II, is equal to $375 r_{0} / u_{j}$ in all cases. After the initial transitory period, density, velocity components and pressure are recorded from time $t=100 r_{0} / u_{j}$ along the jet axis, and on two surfaces at $r=r_{0}$ and $r=r_{c}=6.5 r_{0}$, at a sampling frequency allowing the computation of spectra up to a Strouhal number of $\mathrm{St}_{D}=f D / u_{j}=20$, where $f$ is the time frequency. The cylindrical surface surrounding the jets is located at $r=6.5 r_{0}$ because, as indicated previously, the radial mesh spacing is uniform for $3 r_{0} \leq r \leq 6.75 r_{0}$ but then increases for $r \geq 6.75 r_{0}$. The radial mesh spacing at $r=6.5 r_{0}$ furthermore yields a Strouhal number of $\mathrm{St}_{D}=f D / u_{j}=6.9$ for an acoustic wave discretized by four points per wavelength. In the azimuthal direction, every fourth grid point is stored, allowing data post-processing to be performed up to an azimuthal mode $n_{\theta}=128$, where $n_{\theta}$ is the dimensionless azimuthal wave number such that $n_{\theta}=k_{\theta} r$. The velocity spectra are evaluated from overlapping samples of duration $27.4 r_{0} / u_{j}$. The flow statistics are determined from $t=175 r_{0} / u_{j}$, and they are averaged in the azimuthal direction. They can be considered to be well converged in view of the results obtained at intermediary stages of the LES for $t \geq 300 r_{0} / u_{j}$.

The simulations have been performed using NEC SX-8 computers, on 7 processors using OpenMP, leading to a central processing unit (CPU) speed of around 36 Gflops. Each LES required around $7000 \mathrm{CPU}$ h and $60 \mathrm{~GB}$ of memory for 164000 time steps.

\section{Far-field extrapolation}

The LES near fields are propagated to the acoustic far field by solving the isentropic linearized Euler equations (ILEE) in cylindrical coordinates. ${ }^{35,37,49}$ The extrapolation is performed from fluctuating velocities and pressure recorded in the LES on a surface at $r=6.5 r_{0}$ as mentioned above (see Figure 1(b)). Concerning the position of the surface, it can be noted that very similar far-field results were obtained using two surfaces at $r=5.25 r_{0}$ and at $r=7.25 r_{0}$ in Bogey and Bailly ${ }^{34}$ for an initially laminar jet. The data at $r=6.5 r_{0}$ are interpolated onto a cylindrical surface discretized by an axial mesh spacing of $\Delta z=0.065 r_{0}$. They are then imposed at the bottom boundary of the grid on which the ILEE are solved using the same numerical methods as in the LES. This grid contains $845 \times 256 \times 1155$ points, and extends axially from $z=-16.6 r_{0}$ to $58.2 r_{0}$ and radially up to $r=61.4 r_{0}$. The grid spacings are uniform with $\Delta r=\Delta z=0.065 r_{0}$, yielding $\mathrm{St}_{D}=8.6$ for an acoustic wave at four points per wavelength. After a propagation time of $t \simeq 60 r_{0} / u_{j}$, pressure is recorded around the jets at a distance of $60 r_{0}$ from $z=r=0$, where far-field acoustic conditions are expected to apply according to the experiments of Ahuja et al. ${ }^{50}$ during a period of $250 r_{0} / u_{j}$. Pressure spectra are evaluated using overlapping samples of duration $38 r_{0} / u_{j}$, and they are averaged in the azimuthal direction.

\section{RESULTS}

In this section, the aerodynamic and acoustic results obtained for the jets at $\operatorname{Re}_{D}=5 \times 10^{4}$ are first presented. Those for the jets at $\operatorname{Re}_{\theta} \simeq 480$ are then shown. 
(a)

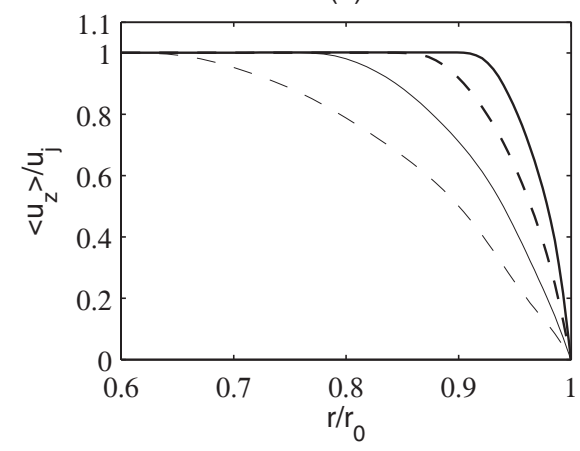

(b)

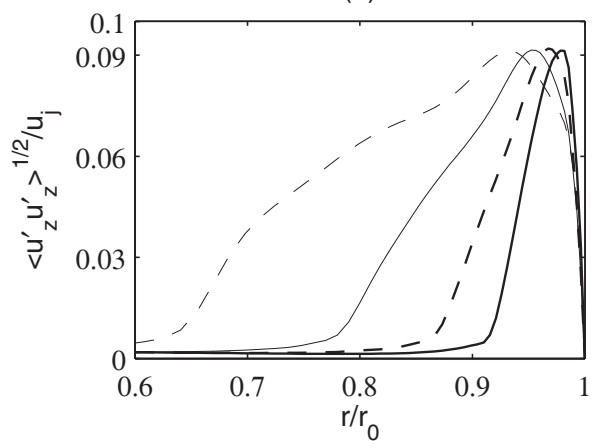

FIG. 2. Profiles at $z=0$ (a) of mean axial velocity $\left\langle u_{z}\right\rangle$ and (b) of the rms values of fluctuating axial velocity $u_{z}^{\prime}$ for the jets at $\operatorname{Re}_{D}=5 \times 10^{4}$ with an inlet boundary-layer thickness (bold solid line) $\delta_{0}=0.09 r_{0}$, (bold dashed line) $\delta_{0}=0.15 r_{0}$, (thin solid line) $\delta_{0}=0.25 r_{0}$, (thin dashed line) $\delta_{0}=0.42 r_{0}$.

\section{A. Jets at $\operatorname{Re}_{D}=5 \times 10^{4}$}

\section{Nozzle-exit conditions}

The profiles of mean and rms axial velocities obtained at the nozzle exit for the four jets at $\operatorname{Re}_{D}$ $=5 \times 10^{4}$ defined in Table I(a) are represented in Figure 2. In Figure 2(a), as intended, the mean velocity profiles are similar to the Blasius laminar profiles imposed at the pipe-nozzle inlet. They are characterized by shape factors between $H=2.25$ and $H=2.30$, and by momentum thicknesses $\delta_{\theta}(0)$ $=0.0119 r_{0}, 0.0191 r_{0}, 0.0308 r_{0}$, and $0.0507 r_{0}$, yielding Reynolds numbers $\operatorname{Re}_{\theta}=304,486,782$, and 1288, as indicated in Table III. The effects of viscosity on the jet mixing layers are consequently expected $^{27}$ to vary significantly with the ratio $\delta_{0} / r_{0}$. On the other hand, they can be considered to be very small on linear instability waves potentially developing in the shear layers according to the work by Morris. ${ }^{21}$ In Figure 2(b), the peak turbulence intensities $u_{e}^{\prime} / u_{j}$ are also observed to be close to $9 \%$ in all cases, see exact values in Table III. The initial flow conditions in the present jets therefore correspond to those found in highly disturbed, or nominally (not fully) turbulent boundary layers. Comparable conditions were measured, for instance, by Batt, ${ }^{51}$ Hussain and Zedan, ${ }^{18}$ and Zaman $^{6,7}$ in tripped shear layers and jets with nozzle-exit parameters $u_{e}^{\prime} / u_{j} \simeq 10 \%$ and $\operatorname{Re}_{\theta}=400$, $u_{e}^{\prime} / u_{j} \simeq 6 \%$ and $184 \leq \operatorname{Re}_{\theta} \leq 349$, and $u_{e}^{\prime} / u_{j} \simeq 9 \%$ and $900 \leq \operatorname{Re}_{\theta} \leq 2250$, respectively.

As in our previous studies, ${ }^{27,35-37}$ the properties of the jet initial disturbances are examined by computing spectra of the fluctuating axial velocity at $r=r_{0}$ at a position close to the nozzle exit in order to avoid the turbulence features being strongly affected by the mixing-layer development. The spectra are calculated at $z=3.3 \delta_{0}$, giving $z=0.3 r_{0}, 0.5 r_{0}, 0.83 r_{0}$, and $1.39 r_{0}$ for the jets with $\delta_{0}$ $=0.09 r_{0}, 0.15 r_{0}, 0.25 r_{0}$, and $0.42 r_{0}$, respectively. They are represented as a function of the Strouhal number $\mathrm{St}_{D}=f D / u_{j}$ in Figure 3(a), and of the azimuthal mode $n_{\theta}$ in Figure 3(b). Their overall shapes are found not to change fundamentally with the exit boundary-layer thickness. For all jets, indeed, the spectra in Figure 3(a) are rather flat for lower Strouhal numbers and then decrease rapidly with

TABLE III. Nozzle-exit conditions for the jets at $\operatorname{Re}_{D}=5 \times 10^{4}$ : peak turbulence intensity $u_{e}^{\prime} / u_{j}$, shape factor $H$ and momentum thickness $\delta_{\theta}(0)$ of the boundary layers, and Reynolds number $\operatorname{Re}_{\theta}$ based on $\delta_{\theta}(0)$.

\begin{tabular}{llllr}
\hline \hline$\delta_{0} / r_{0}$ & $u_{e}^{\prime} / u_{j}$ & $H$ & $\delta_{\theta}(0) / r_{0}$ & $\operatorname{Re}_{\theta}$ \\
\hline 0.09 & $9.14 \%$ & 2.25 & 0.0119 & 304 \\
0.15 & $9.20 \%$ & 2.30 & 0.0191 & 486 \\
0.25 & $9.15 \%$ & 2.26 & 0.0308 & 782 \\
0.42 & $9.12 \%$ & 2.29 & 0.0507 & 1288 \\
\hline \hline
\end{tabular}


(a)

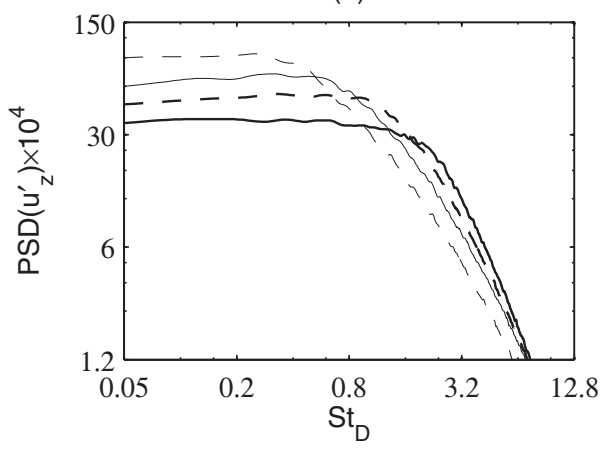

(b)

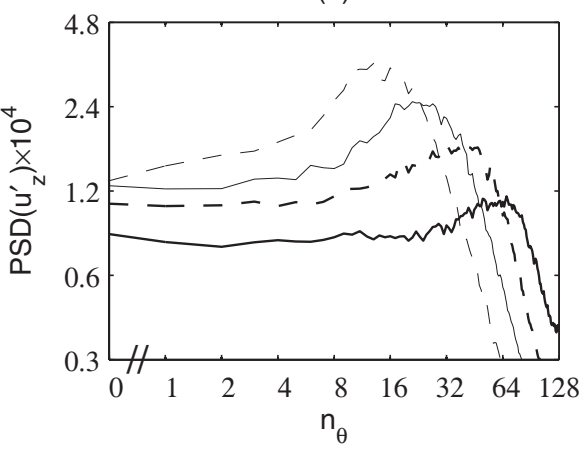

FIG. 3. Power spectral densities (PSD) normalized by $u_{j}$ of axial velocity $u_{z}^{\prime}$ as functions (a) of Strouhal number $\mathrm{St}_{D}=f D / u_{j}$ and (b) of azimuthal mode $n_{\theta}$ for the jets at $\operatorname{Re}_{D}=5 \times 10^{4}$ with (bold solid line) $\delta_{0}=0.09 r_{0}$, (bold dashed line) $\delta_{0}=0.15 r_{0}$, (thin solid line) $\delta_{0}=0.25 r_{0}$, (thin dashed line) $\delta_{0}=0.42 r_{0}$, at $r=r_{0}$ and $z=0.3 r_{0}, z=0.5 r_{0}$, $z=0.83 r_{0}$, and $z=1.39 r_{0}$, respectively.

increasing frequency, while the spectra in Figure 3(b) show a distribution of turbulent energy over a large number of azimuthal modes with peak components between $n_{\theta}=8$ and 64 . As expected, however, they gradually shift towards lower frequencies and modes as the ratio $\delta_{0} / r_{0}$ grows.

In order to take the difference in nozzle-exit boundary-layer thickness into account, the spectra of Figure 3 are re-plotted in Figure 4 versus $\mathrm{St}_{\theta}=f \delta_{\theta}(0) / u_{j}$ and $k_{\theta} \delta_{\theta}(0)$ using a scaling ${ }^{35}$ with $\delta_{\theta}(0)$. The spectra obtained in this way for the four jets strongly resemble each other. In particular, the dominant components in the azimuthal spectra are located around normalized wave numbers $k_{\theta} \delta_{\theta}(0)$ $\simeq 0.8$ (or $k_{\theta} \delta_{0} \simeq 7$ ) in all cases. These results were discussed in a short note, ${ }^{36}$ which also provided comparisons with data available in the literature for turbulent pipe flows ${ }^{52}$ and boundary layers. ${ }^{53}$ Close similarities between the large-scale structures at the nozzle exit of the tripped jets and those in fully developed wall-bounded flows were highlighted. In the present study, the velocity spectra are moreover seen to change slightly as the exit boundary-layer thickness varies. With increasing $\delta_{0} / r_{0}$, the magnitude of the low frequency components, found here for Strouhal numbers $\mathrm{St}_{\theta} \lesssim 0.008$ and azimuthal wave numbers $k_{\theta} \delta_{\theta}(0) \lesssim 0.48$, is reduced, whereas the levels for $\mathrm{St}_{\theta} \gtrsim 0.032$ are higher in Figure 4(a), and those for $k_{\theta} \delta_{\theta}(0) \gtrsim 0.96$ remain roughly the same in Figure 4(b). A larger part of the turbulent energy is thus contributed by fine-scale structures. This trend is in good agreement with

(a)

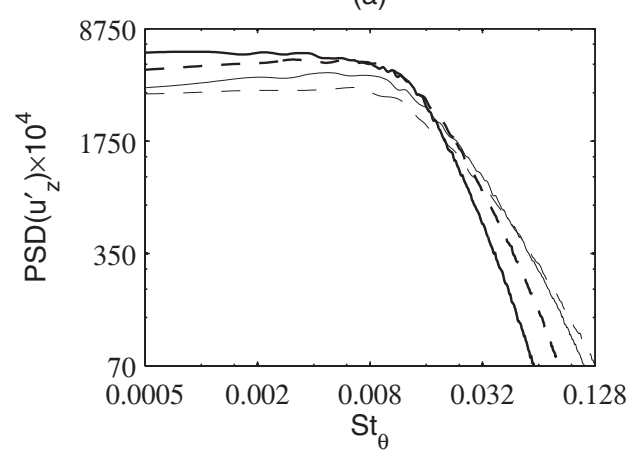

(b)

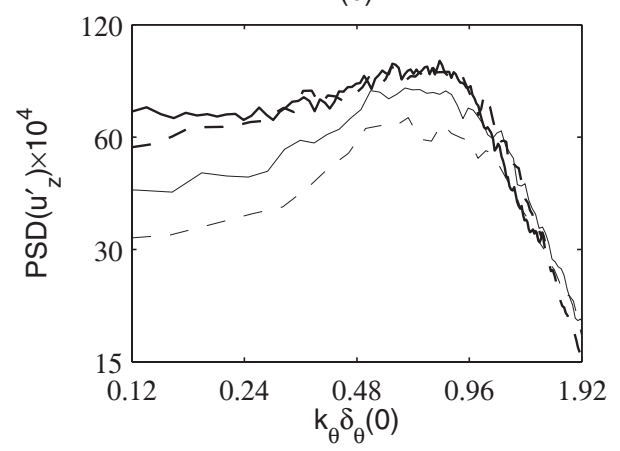

FIG. 4. Power spectral densities (PSD) normalized by $u_{j}$ of velocity $u_{z}^{\prime}$ as functions (a) of Strouhal number $\operatorname{St}_{\theta}=f \delta_{\theta}(0) / u_{j}$ and (b) of azimuthal wave number $k_{\theta} \delta_{\theta}(0)$ for the jets at $\operatorname{Re}_{D}=5 \times 10^{4}$ with (bold solid line) $\delta_{0}=0.09 r_{0}$, (bold dashed line) $\delta_{0}=0.15 r_{0}$, (thin solid line) $\delta_{0}=0.25 r_{0}$, (thin dashed line) $\delta_{0}=0.42 r_{0}$, at $r=r_{0}$ and $z=0.3 r_{0}, z=0.5 r_{0}, z=0.83 r_{0}$, and $z=1.39 r_{0}$, respectively. 
(a)

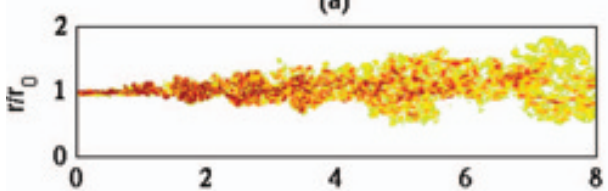

(c)

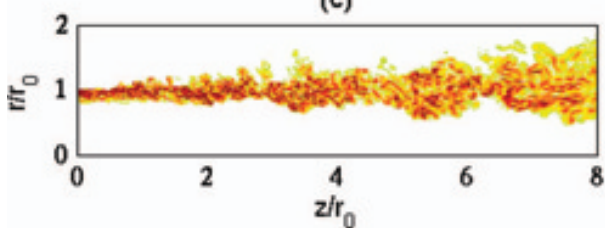

(b)

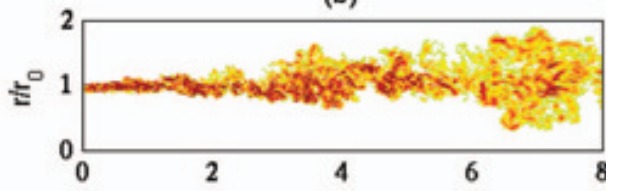

(d)

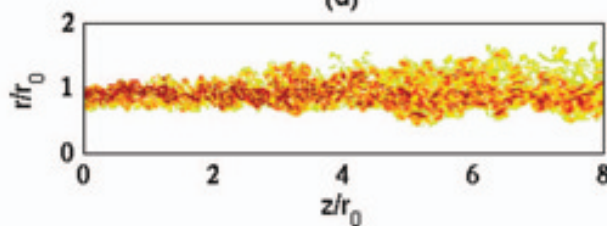

FIG. 5. Snapshots in the $(z, r)$ plane of vorticity norm $|\omega|$ downstream of the nozzle up to $z=8 r_{0}$ for the jets at $\operatorname{Re}_{D}$ $=5 \times 10^{4}$ with (a) $\delta_{0}=0.09 r_{0}$, (b) $\delta_{0}=0.15 r_{0}$, (c) $\delta_{0}=0.25 r_{0}$, (d) $\delta_{0}=0.42 r_{0}$. The color scale ranges up to the level of $17.5 u_{j} / r_{0}, 15.5 u_{j} / r_{0}, 13.5 u_{j} / r_{0}$, and $11.5 u_{j} / r_{0}$, respectively. Only $r \geq 0$ is shown (enhanced online) [URL: http://dx.doi.org/10.1063/1.4807071.1].

that obtained in a recent work ${ }^{27}$ on tripped jets with a fixed $\delta_{0}=0.15 r_{0}$ at Reynolds numbers $\operatorname{Re}_{D}$ between $2.5 \times 10^{4}$ and $2 \times 10^{5}$, and $\operatorname{Re}_{\theta}$ ranging from 256 to 1856 . It can therefore be attributed to viscosity effects as $\operatorname{Re}_{\theta}$ grows with $\delta_{0}$ in the jets.

\section{Vorticity and pressure snapshots}

As first illustrations of the jet flow and noise characteristics, snapshots of the vorticity norm, and fluctuating pressure given by the LES are provided. Vorticity fields obtained up to $z=8 r_{0}$ in the mixing layers are first presented in Figure 5 and Movie 1. The difference in shear-layer thickness appears clearly in the vicinity of the nozzle exit, but is not obvious farther downstream, typically for $z \geq 4 r_{0}$. This suggests a slower development as the ratio $\delta_{0} / r_{0}$ rises.

In order to perform relevant comparisons between the mixing-layer vorticity fields, the axial and radial distances are normalized by the initial momentum thickness $\delta_{\theta}(0)$. The vorticity snapshots obtained up to $z=225 \delta_{\theta}(0)$, corresponding to $z=2.7 r_{0}, 4.3 r_{0}, 6.9 r_{0}$, and $11.4 r_{0}$ with increasing $\delta_{0}$, are shown in Figure 6 and Movie 2. For the jet with $\delta_{0}=0.09 r_{0}$, large-scale structures resembling the coherent vortical structures revealed by the visualizations of Brown and Roshko ${ }^{25}$ are clearly visible in Figure 6(a). As the nozzle-exit boundary layers become thicker, such large-scale structures are, however, more difficult to observe, and small-scale turbulence gradually strengthens. The mixinglayer spreading rate seems also to be reduced, see, for instance, in Figure 6(d) for the jet with $\delta_{0}$

(a)

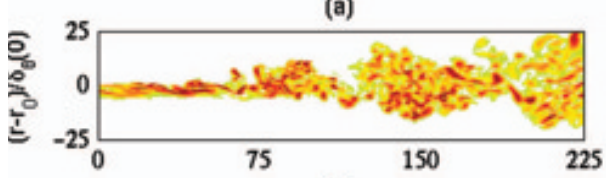

(c)

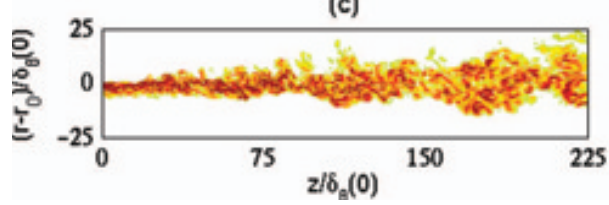

(b)

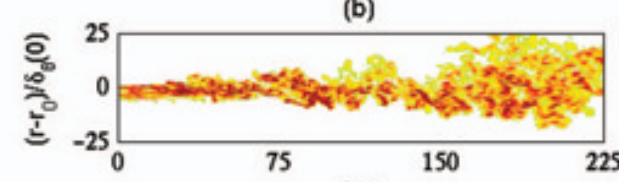

(d)

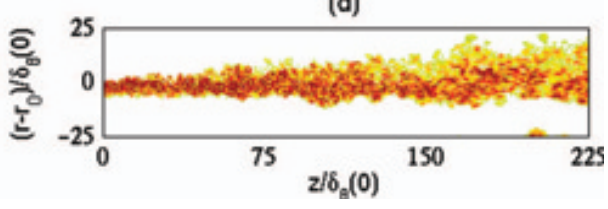

FIG. 6. Snapshots in the $(z, r)$ plane of vorticity norm $|\omega|$, represented using axes normalized by $\delta_{\theta}(0)$, for the jets at $\operatorname{Re}_{D}$ $=5 \times 10^{4}$ with (a) $\delta_{0}=0.09 r_{0}$, (b) $\delta_{0}=0.15 r_{0}$, (c) $\delta_{0}=0.25 r_{0}$, (d) $\delta_{0}=0.42 r_{0}$. The color scale ranges up to the level of $0.42 u_{j} / \delta_{\theta}(0)$ (enhanced online) [URL: http://dx.doi.org/10.1063/1.4807071.2]. 


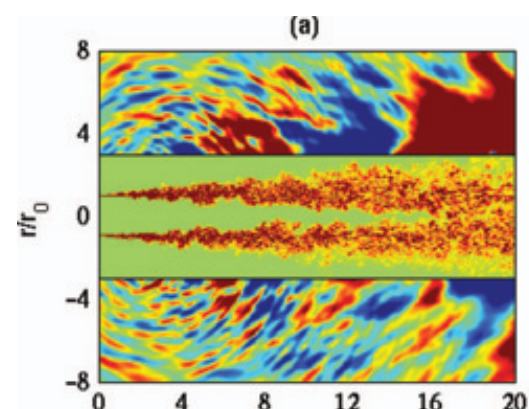

(c)

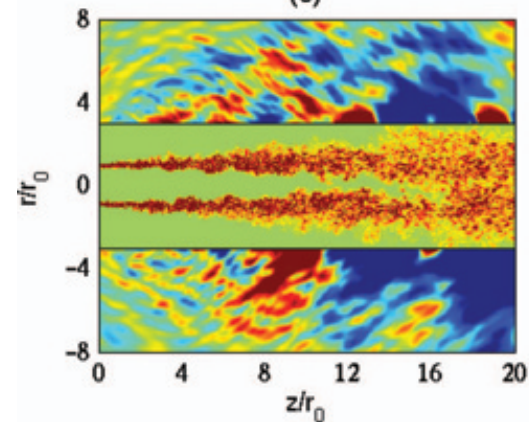

(b)

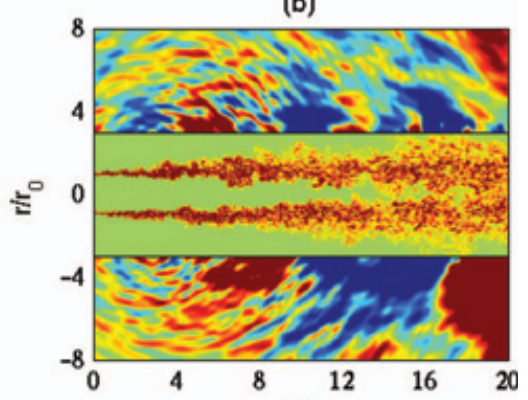

(d)

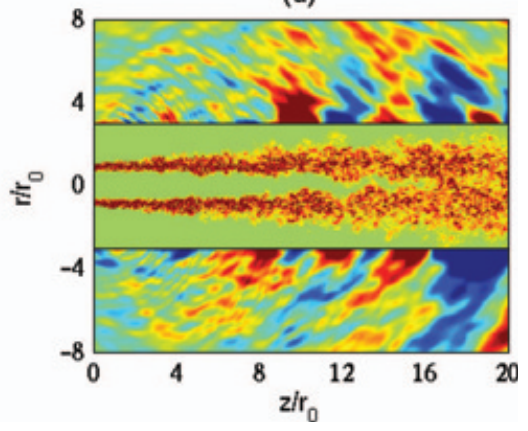

FIG. 7. Snapshots in the $(z, r)$ plane of vorticity norm $|\omega|$ in the flow and of fluctuating pressure $p-p_{a}$ outside, for the jets at $\operatorname{Re}_{D}=5 \times 10^{4}$ with (a) $\delta_{0}=0.09 r_{0}$, (b) $\delta_{0}=0.15 r_{0}$, (c) $\delta_{0}=0.25 r_{0}$, (d) $\delta_{0}=0.42 r_{0}$. The color scales range up to the level of $5.5 u_{j} / r_{0}$ for the vorticity, and from -70 to $70 \mathrm{~Pa}$ for the pressure (enhanced online) [URL: http://dx.doi.org/10.1063/1.4807071.3].

$=0.42 r_{0}$. Similar observations were made in previous simulations ${ }^{27}$ of tripped jets with increasing Reynolds numbers $\operatorname{Re}_{D}$ and $\operatorname{Re}_{\theta}$.

Snapshots of the vorticity norm up to $z=20 r_{0}$ in the jets, and of the near-field pressure fluctuations outside are displayed in Figure 7 and Movie 3. The jet overall developments are not seen to differ fundamentally. The sound field, on the contrary, changes appreciably with the initial shear-layer thickness in terms of both structure and level. Compare, for example, Figures 7(a) and $7(\mathrm{~d})$. In the jet with $\delta_{0}=0.09 r_{0}$, strong acoustic waves are generated in the mixing layers around $z=3 r_{0}$. This is visibly not the case in the jet with $\delta_{0}=0.42 r_{0}$, for which the emitted noise appears much weaker. Comparable modifications of the sound field features were noticed in our LES ${ }^{27}$ recently performed to study the Reynolds number effects in jets.

\section{Shear-layer development}

To quantify the influence of the ratio $\delta_{0} / r_{0}$ on the shear layers of the present jets at $\operatorname{Re}_{D}=5 \times 10^{4}$, the variations between $z=0$ and $z=10 r_{0}$ of the momentum thickness $\delta_{\theta}$ and of the maximum rms values of the axial velocity $u_{z}^{\prime}$ are presented in Figure 8 . As the nozzle-exit boundary-layer thickness increases, the mixing layers are found to develop more slowly in Figure 8(a), resulting in curves of $\delta_{\theta}$ crossing each other, hence in smaller shear-layer thickness for larger $\delta_{0}$ farther downstream. At the same time, reduced turbulence levels are obtained in Figure 8(b). The rms axial velocity profiles reach peak values of $0.171 u_{j}$ for $\delta_{0}=0.09 r_{0}, 0.164 u_{j}$ for $\delta_{0}=0.15 r_{0}, 0.154 u_{j}$ for $\delta_{0}=0.25 r_{0}$, and only $0.148 u_{j}$ for $\delta_{0}=0.42 r_{0}$, refer to Table IV for other velocity components. They also show an overshoot around $z=1.5 r_{0}$ and $z=3.5 r_{0}$ for $\delta_{0}=0.09 r_{0}$ and $\delta_{0}=0.15 r_{0}$, respectively, whereas they grow nearly monotonically in the two other cases. The shear-layer transition is consequently smoother in the jets with thicker exit boundary layers.

Using a scaling with the initial shear-layer momentum thickness $\delta_{\theta}(0)$, the variations of $\delta_{\theta} / \delta_{\theta}(0)$ and of the maximum rms values of $u_{z}^{\prime}$ are plotted in Figures 9 (a) and 9(b) as a function of $z / \delta_{\theta}(0)$ 
(a)

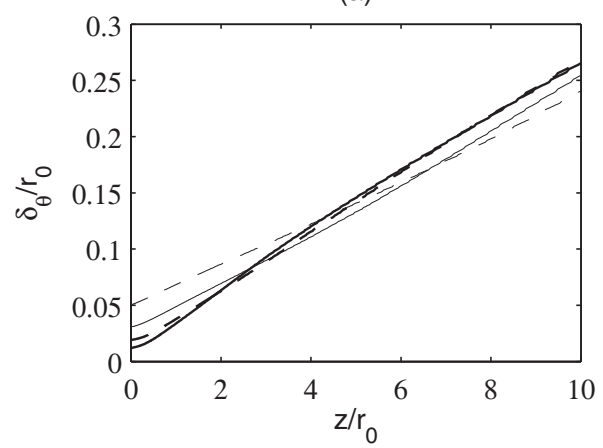

(b)

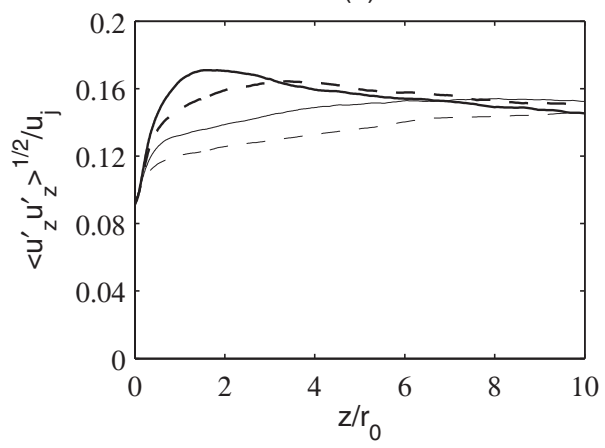

FIG. 8. Variations with $z / r_{0}$ (a) of shear-layer momentum thickness $\delta_{\theta}$ and (b) of peak rms values of fluctuating axial velocity $u_{z}^{\prime}$ for the jets at $\operatorname{Re}_{D}=5 \times 10^{4}$ with (bold solid line) $\delta_{0}=0.09 r_{0}$, (bold dashed line) $\delta_{0}=0.15 r_{0}$, (thin solid line) $\delta_{0}=0.25 r_{0}$, (thin dashed line) $\delta_{0}=0.42 r_{0}$.

from $z=0$ up to $z=250 \delta_{\theta}(0)$. The profiles clearly indicate that the mixing-layer spreading rates and the turbulence intensities both decrease with increasing $\delta_{0}$. They are very similar to those obtained in a recent computational study ${ }^{27}$ for axisymmetric shear layers with initial parameters $u_{e}^{\prime} / u_{j} \simeq 9 \%$ and $\operatorname{Re}_{\theta}$ rising from 256 to 1856 . With Reynolds numbers $\operatorname{Re}_{\theta}$ ranging from 304 for $\delta_{0}=0.09 r_{0}$ up to 1288 for $\delta_{0}=0.42 r_{0}$, the present results are therefore most probably due to Reynolds number effects, as mentioned above.

To give additional information about the shear-layer turbulence, the variations of the axial integral length scales $L_{u u}^{(z)}$ evaluated from velocity $u_{z}^{\prime}$ along the lip line are presented as a function of $z / r_{0}$ in Figure 10(a) and of $z / \delta_{\theta}(0)$ in Figure 10(b). After a decrease shortly downstream of the nozzle exit, the length scales are noted to grow fairly linearly, which is in agreement with experimental data of the literature.$^{54,55}$ More interestingly, when they are scaled by $\delta_{\theta}(0)$ as in Figure 10(b), they appear persistently smaller in initially thicker mixing layers. This result can naturally be attributed to the increase of the Reynolds number $\mathrm{Re}_{\theta}$, leading to a strengthening of fine-scale structures according to our past work ${ }^{27}$ and the illustrations below.

Finally, spectra of the radial velocity $u_{r}^{\prime}$ are computed on the lip line at $z=10 \delta_{0}$, providing $z=0.9 r_{0}, 1.5 r_{0}, 2.5 r_{0}$, and $4.2 r_{0}$ for the jets with $\delta_{0}=0.09 r_{0}, 0.15 r_{0}, 0.25 r_{0}$, and $0.42 r_{0}$, respectively. They are plotted as a function of $\mathrm{St}_{D}$ in Figure 11(a) and of $\mathrm{St}_{\theta}$ in Figure 11(b). With increasing initial shear-layer thickness, the dominant components shift towards lower diameter-based Strouhal numbers, as expected. However, when the spectra are represented versus $\mathrm{St}_{\theta}$, they remain centered around $\mathrm{St}_{\theta} \simeq 0.012$. This value corresponds to the frequencies predominating early on in initially laminar annular mixing layers according to experiments ${ }^{23}$ and to the linear stability analyses conducted by Morris ${ }^{21,22}$ and Michalke, ${ }^{19}$ and in the Appendix. This indicates that an instability-like component emerges in the highly disturbed shear layers of the present jets. Nevertheless, as the exit boundary-layer thickness and thence $\mathrm{Re}_{\theta}$ are larger, the velocity spectra broaden and this peak component becomes less distinct.

TABLE IV. Peak values of turbulence intensities in the jets at $\operatorname{Re}_{D}=5$ $\times 10^{4}$.

\begin{tabular}{ccccc}
\hline \hline$\delta_{0} / r_{0}$ & $\left\langle u_{z}^{\prime 2}\right\rangle^{1 / 2} / u_{j}$ & $\left\langle u_{r}^{\prime 2}\right\rangle^{1 / 2} / u_{j}$ & $\left\langle u_{\theta}^{\prime 2}\right\rangle^{1 / 2} / u_{j}$ & $\left\langle u_{r}^{\prime} u_{z}^{\prime}\right\rangle^{1 / 2} / u_{j}$ \\
\hline 0.09 & $17.1 \%$ & $13.3 \%$ & $15.0 \%$ & $11.0 \%$ \\
0.15 & $16.4 \%$ & $12.3 \%$ & $13.9 \%$ & $10.1 \%$ \\
0.25 & $15.4 \%$ & $11.3 \%$ & $13.0 \%$ & $9.3 \%$ \\
0.42 & $14.8 \%$ & $10.7 \%$ & $12.3 \%$ & $8.8 \%$ \\
\hline \hline
\end{tabular}


(a)

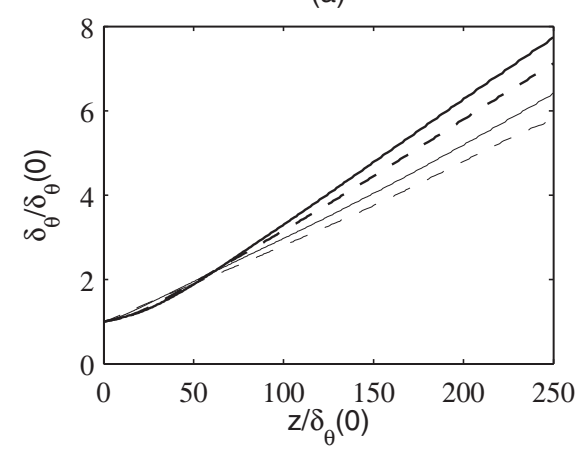

(b)

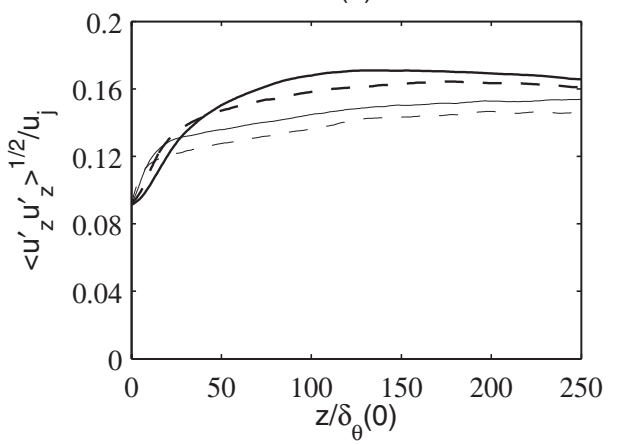

FIG. 9. Variations with $z / \delta_{\theta}(0)$ (a) of shear-layer momentum thickness $\delta_{\theta} / \delta_{\theta}(0)$ and (b) of peak rms values of velocity $u_{z}^{\prime}$ for the jets at $\operatorname{Re}_{D}=5 \times 10^{4}$ with (bold solid line) $\delta_{0}=0.09 r_{0}$, (bold dashed line) $\delta_{0}=0.15 r_{0}$, (thin solid line) $\delta_{0}=0.25 r_{0}$, (thin dashed line) $\delta_{0}=0.42 r_{0}$.

\section{Jet development}

The variations of the mean and rms axial velocities on the jet axis are presented in Figure 12. Measurements ${ }^{4,56,57}$ available for Mach number 0.9 jets at high Reynolds numbers $\operatorname{Re}_{D} \geq 5 \times 10^{5}$, which can be expected to be initially turbulent, are also depicted for the comparison. Increasing the initial shear-layer thickness appears in Figure 12(a) to reduce the potential core length from $z_{c}=15.8 r_{0}$ for $\delta_{0}=0.09 r_{0}$ down to $z_{c}=13.7 r_{0}$ for $\delta_{0}=0.42 r_{0}$, where $z_{c}$ is arbitrarily defined by $\left\langle u_{z}\right\rangle\left(z=z_{c}\right)=0.95 u_{j}$, as reported in Table V. It seems also to result in a more rapid velocity decay downstream of the potential core. Regarding the centerline axial turbulence intensities in Figure 12(b), they are similar with peak values around $0.115 u_{j}$, except for the jet with $\delta_{0}=0.25 r_{0}$ in which the peak value is higher and equal to $0.136 u_{j}$, refer to Table $\mathrm{V}$ for the radial velocity component showing a similar trend. The centerline velocity profiles moreover correspond fairly well to the measurements, which are obtained for jets with differing, and incompletely specified, exit flow conditions.

The centerline profiles of mean and turbulent axial velocities are re-plotted in Figure 13 as a function of $z-z_{c}$. The influence of the nozzle-exit boundary-layer thickness on the jet development downstream of the potential core thus turns out to be relatively limited. Thicker initial shear layers,

(a)

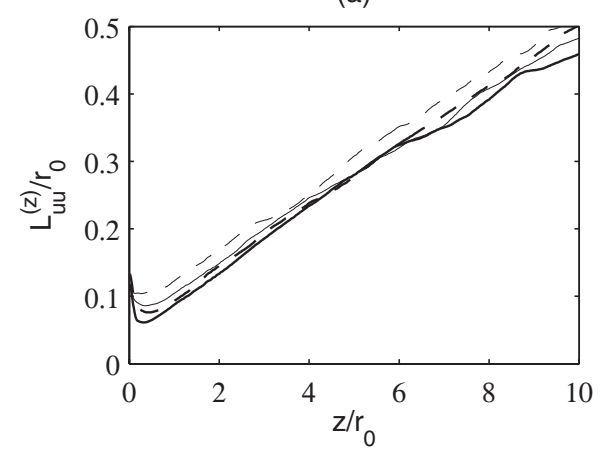

(b)

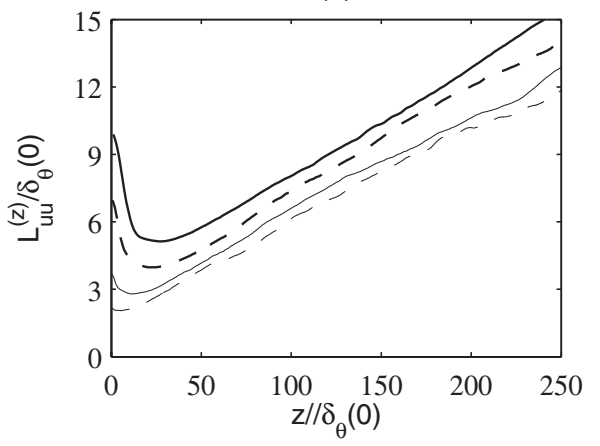

FIG. 10. Variations of axial integral length scales, (a) $L_{u u}^{(z)} / r_{0}$ with $z / r_{0}$ and (b) $L_{u u}^{(z)} / / \delta_{\theta}(0)$ with $z / \delta_{\theta}(0)$, calculated from velocity $u_{z}^{\prime}$ at $r=r_{0}$ for the jets at $\operatorname{Re}_{D}=5 \times 10^{4}$ with (bold solid line) $\delta_{0}=0.09 r_{0}$, (bold dashed line) $\delta_{0}=0.15 r_{0}$, (thin solid line) $\delta_{0}=0.25 r_{0}$, (thin dashed line) $\delta_{0}=0.42 r_{0}$. 
(a)

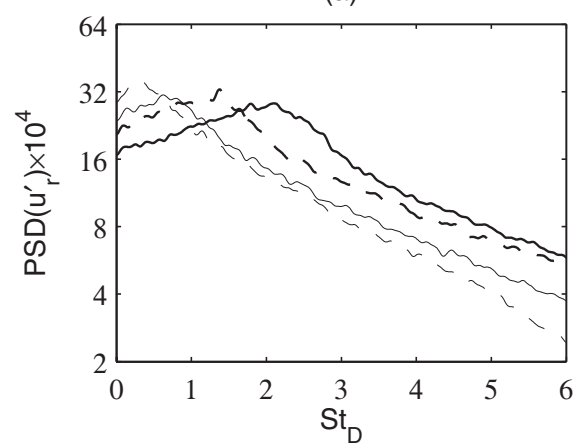

(b)

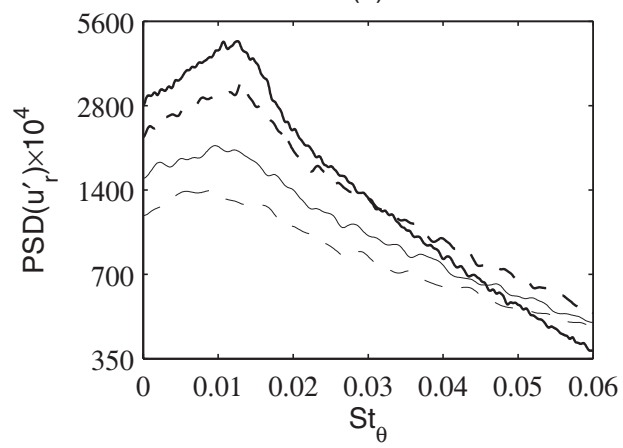

FIG. 11. Power spectral densities (PSD) normalized by $u_{j}$ of radial velocity $u_{r}^{\prime}$ as functions of Strouhal numbers (a) $\mathrm{St}_{D}=f D / u_{j}$ and (b) $\mathrm{St}_{\theta}=f \delta_{\theta}(0) / u_{j}$ for the jets at $\operatorname{Re}_{D}=5 \times 10^{4}$ with (bold solid line) $\delta_{0}=0.09 r_{0}$, (bold dashed line) $\delta_{0}=0.15 r_{0}$, (thin solid line) $\delta_{0}=0.25 r_{0}$, (thin dashed line) $\delta_{0}=0.42 r_{0}$, at $r=r_{0}$ and $z=0.9 r_{0}, z=1.5 r_{0}, z=2.5 r_{0}$, and $z=4.17 r_{0}$, respectively.

(a)

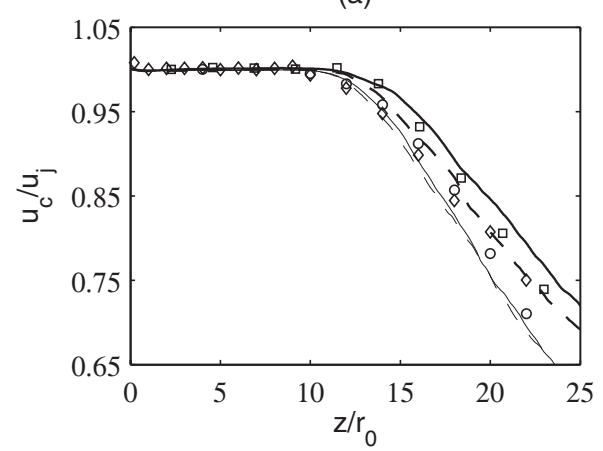

(b)

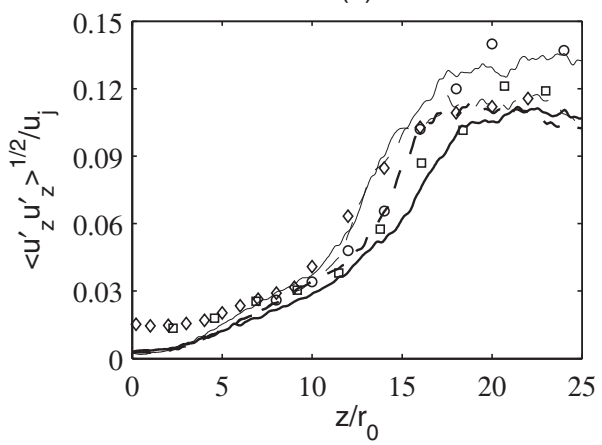

FIG. 12. Variations (a) of centerline mean axial velocity $u_{c}$ and (b) of centerline rms values of velocity $u_{z}^{\prime}$ for the jets at $\operatorname{Re}_{D}$ $=5 \times 10^{4}$ with (bold solid line) $\delta_{0}=0.09 r_{0}$, (bold dashed line) $\delta_{0}=0.15 r_{0}$, (thin solid line) $\delta_{0}=0.25 r_{0}$, (thin dashed line) $\delta_{0}=0.42 r_{0}$. Measurements for jets at $\mathrm{M}=0.9$ and $\operatorname{Re}_{D} \geq 5 \times 10^{5}$ : (circle) Lau et al. ${ }^{56}$ (square) Arakeri et al., ${ }^{4}$ (diamond) Fleury et al. ${ }^{57}$

TABLE V. Axial position of the end of the potential core $z_{c}$, and peak rms values of fluctuating velocities $u_{z}^{\prime}$ and $u_{r}^{\prime}$ on the axis for the jets at $\operatorname{Re}_{D}=5$ $\times 10^{4}$.

\begin{tabular}{cccc}
\hline \hline$\delta_{0} / r_{0}$ & $z_{c} / r_{0}$ & $\left\langle u_{z}^{\prime 2}\right\rangle^{1 / 2} / u_{j}$ & $\left\langle u_{r}^{\prime 2}\right\rangle^{1 / 2} / u_{j}$ \\
\hline 0.09 & 15.8 & $11.1 \%$ & $9.2 \%$ \\
0.15 & 14.7 & $11.4 \%$ & $9.4 \%$ \\
0.25 & 14.1 & $13.6 \%$ & $10.4 \%$ \\
0.42 & 13.7 & $11.7 \%$ & $9.7 \%$ \\
\hline \hline
\end{tabular}


(a)

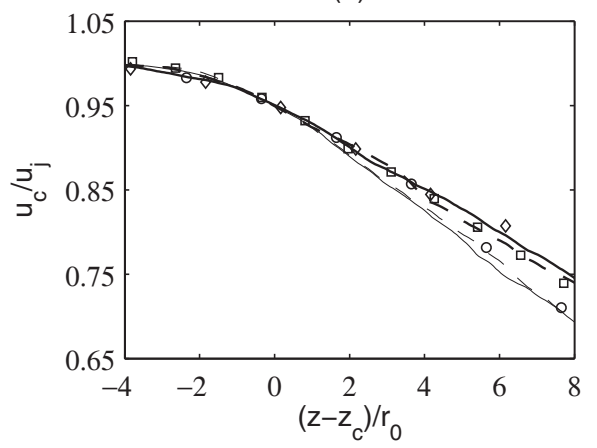

(b)

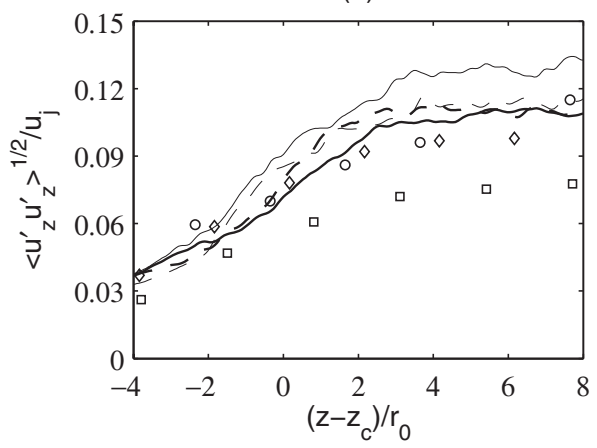

FIG. 13. Variations (a) of centerline mean axial velocity $u_{c}$ and (b) of centerline rms values of velocity $u_{z}^{\prime}$, as a function of $\left(z-z_{c}\right) / r_{0}$, for the jets at $\operatorname{Re}_{D}=5 \times 10^{4}$ with (bold solid line) $\delta_{0}=0.09 r_{0}$, (bold dashed line) $\delta_{0}=0.15 r_{0}$, (thin solid line) $\delta_{0}=0.25 r_{0}$, (thin dashed line) $\delta_{0}=0.42 r_{0}$. Measurements for jets at $\mathrm{M}=0.9$ and $\operatorname{Re}_{D} \geq 5 \times 10^{5}$ : (circle) Lau et al. ${ }^{56}$ (square) Arakeri et al. ${ }^{4}$ (diamond) Fleury et al. ${ }^{57}$

however, leads to a slightly faster velocity decay, and higher turbulence levels are found for the jet with $\delta_{0}=0.25 r_{0}$.

\section{Acoustic fields}

The main properties of the acoustic fields computed at 60 radii from the nozzle exit using the wave extrapolation method described in Sec. II D are investigated. The sound pressure levels obtained in this way are displayed in Figure 14. The jets with thicker initial shear layers are found to generate lower noise. The decrease of the sound levels is particularly significant for the jet with $\delta_{0}=0.42 r_{0}$, with differences of -2.3 and $-2.9 \mathrm{~dB}$ with respect to the jet with $\delta_{0}=0.09 r_{0}$ at the radiation angles $\phi=40^{\circ}$ and $\phi=90^{\circ}$, respectively, see in Table VI. This leads for the former jet to a good agreement with measurements ${ }^{58-60}$ available for Mach number 0.9 jets at $\operatorname{Re}_{D} \geq 5 \times 10^{5}$. The present noise reduction is most probably due to the lowering of the turbulence intensities and to the weakening of large-scale structures in the mixing layers displayed in Figures 8(b) and 11(b), both of which result ${ }^{27}$ from the increase of the Reynolds number $\operatorname{Re}_{\theta}$ from 304 for $\delta_{0}=0.09 r_{0}$ to 1288 for $\delta_{0}=0.42 r_{0}$. It can be pointed out, for the first time as far as we know, that thickening the initial shear layers in jets at a fixed $\mathrm{Re}_{D}$ may cause contrary results on the sound field depending on

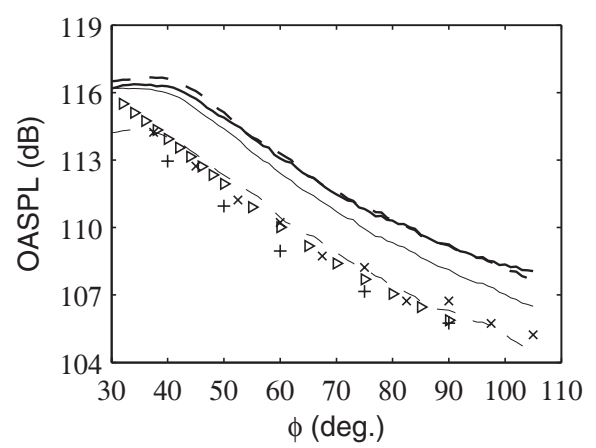

FIG. 14. Overall sound pressure levels (OASPL) at $60 r_{0}$ from the nozzle exit, as a function of the angle $\phi$ relative to the jet direction, for the jets at $\operatorname{Re}_{D}=5 \times 10^{4}$ with (bold solid line) $\delta_{0}=0.09 r_{0}$, (bold dashed line) $\delta_{0}=0.15 r_{0}$, (thin solid line) $\delta_{0}$ $=0.25 r_{0}$, (thin dashed line) $\delta_{0}=0.42 r_{0}$. Measurements for jets at $\operatorname{Re}_{D} \geq 5 \times 10^{5}$ : (plus) Mollo-Christensen et al., ${ }^{58}$ (times) Lush, ${ }^{59}$ (right-pointing triangle) Bogey et al..$^{60}$ 
TABLE VI. Overall sound pressure levels at $60 r_{0}$ from the nozzle exit at $\phi=40^{\circ}$ and $\phi=90^{\circ}$ for the jets at $\operatorname{Re}_{D}=5 \times 10^{4}$.

\begin{tabular}{lll}
\hline \hline$\delta_{0} / r_{0}$ & $\phi=40^{\circ}$ & $\phi=90^{\circ}$ \\
\hline 0.09 & $116.3 \mathrm{~dB}$ & $109.2 \mathrm{~dB}$ \\
0.15 & $116.6 \mathrm{~dB}$ & $109.2 \mathrm{~dB}$ \\
0.25 & $116.0 \mathrm{~dB}$ & $108.1 \mathrm{~dB}$ \\
0.42 & $114.0 \mathrm{~dB}$ & $106.3 \mathrm{~dB}$ \\
\hline \hline
\end{tabular}

the laminar or turbulent flow state at the nozzle exit. Indeed, the noise levels become stronger for fully laminar initial conditions, ${ }^{34}$ but weaker for highly disturbed initial conditions as in this study.

The pressure spectra calculated at $60 r_{0}$ from the nozzle exit at the angles $\phi=40^{\circ}$ and $90^{\circ}$ are given in Figure 15 as a function of the Strouhal number $\mathrm{St}_{D}$, and compared with experimental data for jets at high Reynolds numbers. ${ }^{60,61}$ As the initial shear-layer thickness increases, the amplitude of high-frequency noise components is noticeably reduced. The jet with $\delta_{0}=0.42 r_{0}$ thus emits lower acoustic levels with respect to the other jets down to Strouhal numbers $\mathrm{St}_{D}=0.1$ at $\phi=40^{\circ}$ and $\mathrm{St}_{D}=0.3$ at $\phi=90^{\circ}$. At $\phi=90^{\circ}$, compared to the case with $\delta_{0}=0.09 r_{0}$, the decrease is, for example, around $-3 \mathrm{~dB}$ at $\mathrm{St}_{D}=0.8$ and $-10 \mathrm{~dB}$ at $\mathrm{St}_{D}=3.2$.

\section{B. Jets at $\operatorname{Re}_{\theta} \simeq 480$}

\section{Nozzle-exit conditions}

The profiles of mean and rms axial velocities obtained at $z=0$ for the jets at $\operatorname{Re}_{\theta} \simeq 480$ defined in Table I(b) are presented in Figures 16(a) and 16(b). They are nearly identical to those shown in Figures 2(a) and 2(b), indicating that the nozzle-exit conditions in the jets at $\operatorname{Re}_{D}=5 \times 10^{4}$ and at $\operatorname{Re}_{\theta} \simeq 480$ can be considered as effectively the same except for the Reynolds numbers. As reported in Table VII, the exit boundary layers are characterized by shape factors $2.22 \leq H \leq 2.32$, peak turbulence intensities $9.12 \% \leq u_{e}^{\prime} / u_{j} \leq 9.20 \%$, and momentum thicknesses $\delta_{\theta}(0)=0.0115 r_{0}$, $0.0191 r_{0}, 0.0313 r_{0}$, and $0.0515 r_{0}$. The momentum Reynolds numbers in the jets are consequently very similar, as desired, and lie within the range $471 \leq \mathrm{Re}_{\theta} \leq 487$. In this way, the differences in mixing-layer properties which will be observed in what follows cannot be attributed to Reynolds number effects, but only to the variations of the ratio $\delta_{0} / r_{0}$.

(a)

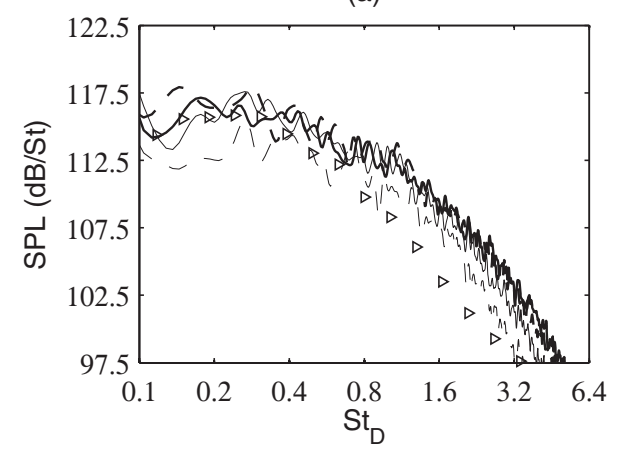

(b)

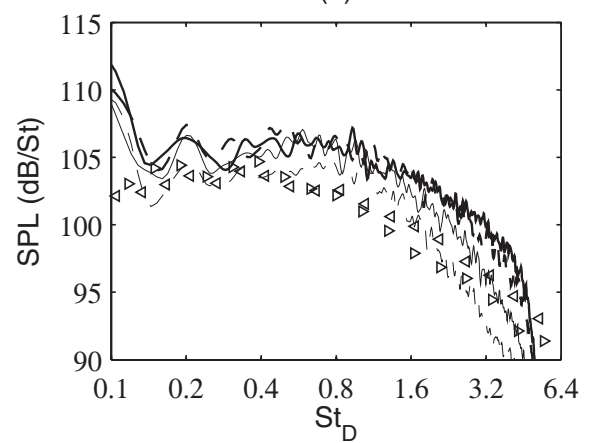

FIG. 15. Sound pressure levels (SPL) at $60 r_{0}$ from the nozzle exit, as a function of $\mathrm{St}_{D}$, at the angles $\phi$ of (a) $40^{\circ}$ and (b) $90^{\circ}$ for the jets at $\operatorname{Re}_{D}=5 \times 10^{4}$ with (bold solid) $\delta_{0}=0.09 r_{0}$, (bold dashed line) $\delta_{0}=0.15 r_{0}$, (thin solid line) $\delta_{0}=0.25 r_{0}$, (thin dashed line) $\delta_{0}=0.42 r_{0}$. Measurements for jets at $\operatorname{Re}_{D} \geq 7.8 \times 10^{5}$ : (left-pointing triangle) Tanna, ${ }^{61}$ (right-pointing triangle) Bogey et al. ${ }^{60}$ 
(a)

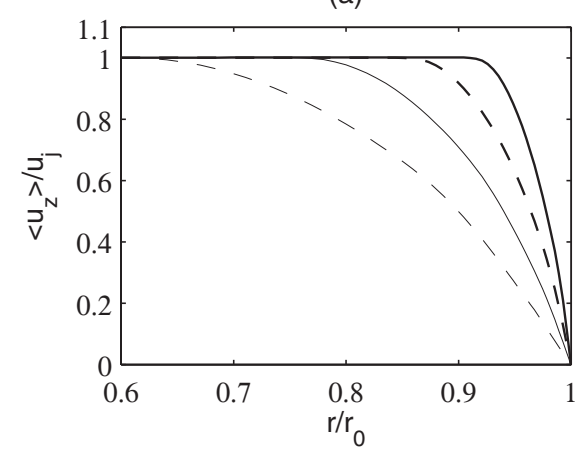

(b)

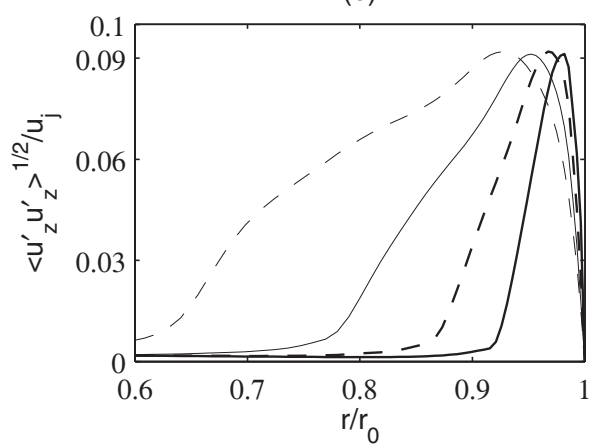

FIG. 16. Profiles at $z=0$ (a) of mean axial velocity $<u_{z}>$ and (b) of the rms values of fluctuating axial velocity $u_{z}^{\prime}$ for the jets at $\operatorname{Re}_{\theta} \simeq 480$ with an inlet boundary-layer thickness (bold solid line) $\delta_{0}=0.09 r_{0}$, (bold dashed line) $\delta_{0}=0.15 r_{0}$, (thin solid line) $\delta_{0}=0.25 r_{0}$, (thin dashed line) $\delta_{0}=0.42 r_{0}$.

As in Sec. III A 1, spectra of axial velocity $u_{z}^{\prime}$ are calculated just downstream of the nozzle lip at $r=r_{0}$ and $z=3.3 \delta_{0}$. They are represented as a function of the Strouhal number $\mathrm{St}_{D}$ in Figure 17(a) and of the azimuthal mode $n_{\theta}$ in Figure 17(b). Their shapes and variations with the nozzle-exit boundary-layer thickness resemble those obtained in Figure 3 for the jets at $\operatorname{Re}_{D}=5$ $\times 10^{4}$.

To identify the possible effects of the boundary-layer thickness in this case, the velocity spectra are plotted in Figure 18 using a scaling with $\delta_{\theta}(0)$. Contrary to what is found in Figure 4 for the jets at a fixed $\mathrm{Re}_{D}$, they nearly superimpose, demonstrating that the properties of the initial disturbances in the present mixing layers with approximately the same $\operatorname{Re}_{\theta}$ does not depend on the ratio $\delta_{0} / r_{0}$. Furthermore, since the boundary layers are better discretized as $\delta_{0} / r_{0}$ increases, this supports that the turbulent transition inside the nozzle pipe is very little sensitive to the grid.

\section{Vorticity and pressure snapshots}

Snapshots of the vorticity norm obtained up to $z=8 r_{0}$ in the shear layers are displayed in Figure 19. Compare, for instance, Figure 19(a) for $\delta_{0}=0.09 r_{0}$ and Figure 19(d) for $\delta_{0}=0.42 r_{0}$. It seems, contrary to what was noted for the jets at $\operatorname{Re}_{D}=5 \times 10^{4}$ in Figure 5, that mixing layers with smaller thickness at the nozzle exit remain thinner than the others farther downstream, which will be checked later.

As previously, the vorticity fields in the shear layers are also represented using $x$ and $y$-axes normalized by the initial momentum thickness $\delta_{\theta}(0)$. The four snapshots obtained up to $z=225 \delta_{\theta}(0)$ are shown in Figure 20. They look very much alike, regardless of $\delta_{0} / r_{0}$, which is expected given that the Reynolds numbers $\operatorname{Re}_{\theta}$ are nearly identical. In particular, unlike the findings observed in Figure 6 for the jets at $\operatorname{Re}_{D}=5 \times 10^{4}$, there is no notable change in the mixing-layer spreading rate, nor in the distribution between large and small turbulent scales. On the latter point, large-scale coherent-like vortical structures are visible in all cases.

TABLE VII. Nozzle-exit conditions for the jets at $\operatorname{Re}_{\theta} \simeq 480$ : peak turbulence intensity $u_{e}^{\prime} / u_{j}$, shape factor $H$ and momentum thickness $\delta_{\theta}(0)$ of the boundary layers, and Reynolds number $\operatorname{Re}_{\theta}$ based on $\delta_{\theta}(0)$.

\begin{tabular}{ccccc}
\hline \hline$\delta_{0} / r_{0}$ & $u_{e}^{\prime} / u_{j}$ & $H$ & $\delta_{\theta}(0) / r_{0}$ & $\operatorname{Re}_{\theta}$ \\
\hline 0.09 & $9.13 \%$ & 2.32 & 0.0115 & 487 \\
0.15 & $9.20 \%$ & 2.30 & 0.0191 & 486 \\
0.25 & $9.12 \%$ & 2.22 & 0.0313 & 477 \\
0.42 & $9.19 \%$ & 2.25 & 0.0515 & 471 \\
\hline \hline
\end{tabular}


(a)

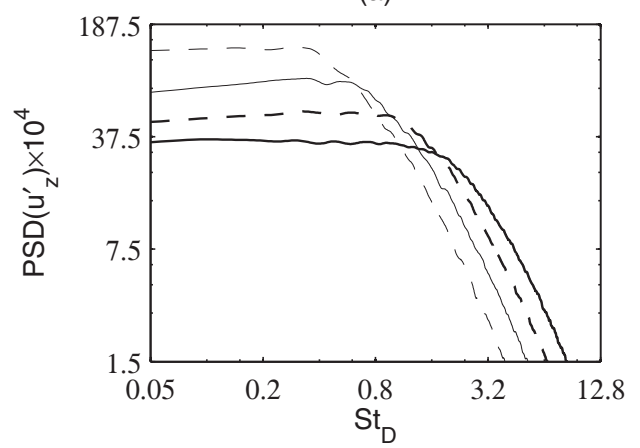

(b)

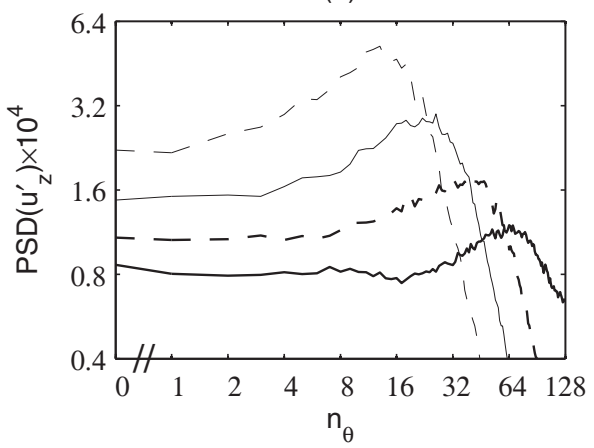

FIG. 17. Power spectral densities (PSD) normalized by $u_{j}$ of axial velocity $u_{z}^{\prime}$ as functions (a) of Strouhal number $\mathrm{St}_{D}=f D / u_{j}$ and (b) of azimuthal mode $n_{\theta}$ for the jets at $\operatorname{Re}_{\theta} \simeq 480$ with (bold solid line) $\delta_{0}=0.09 r_{0}$, (bold dashed line) $\delta_{0}=0.15 r_{0}$, (thin solid line) $\delta_{0}=0.25 r_{0}$, (thin dashed line) $\delta_{0}=0.42 r_{0}$, at $r=r_{0}$ and $z=0.3 r_{0}, z=0.5 r_{0}, z=0.83 r_{0}$, and $z=1.39 r_{0}$, respectively.

(a)

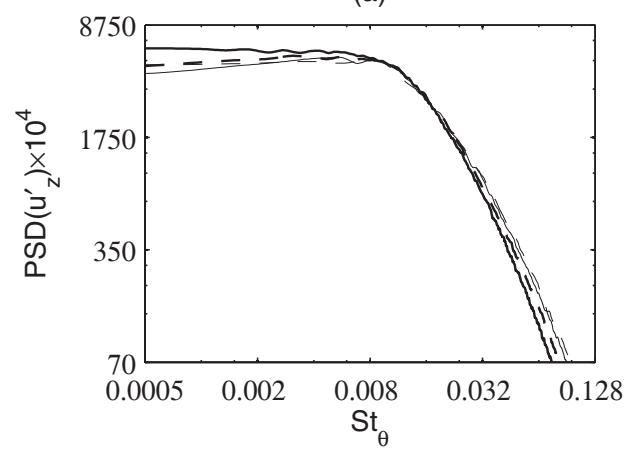

(b)

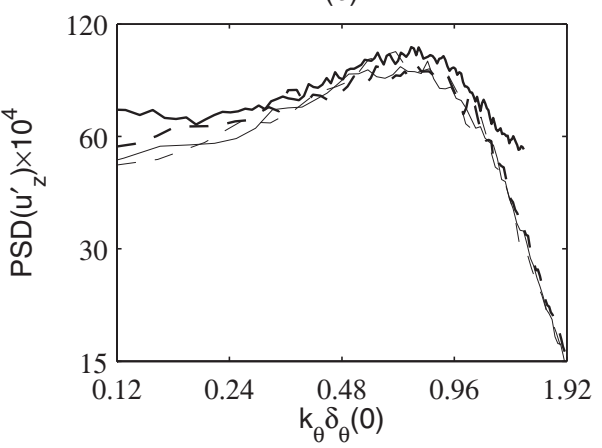

FIG. 18. Power spectral densities (PSD) normalized by $u_{j}$ of velocity $u_{z}^{\prime}$ as functions (a) of Strouhal number $\mathrm{St}_{\theta}=f \delta_{\theta}(0) / u_{j}$ and (b) of azimuthal wave number $k_{\theta} \delta_{\theta}(0)$ for the jets at $\operatorname{Re}_{\theta} \simeq 480$ with (bold solid line) $\delta_{0}=0.09 r_{0}$, (bold dashed line) $\delta_{0}$ $=0.15 r_{0}$, (thin solid line) $\delta_{0}=0.25 r_{0}$, (thin dashed line) $\delta_{0}=0.42 r_{0}$, at $r=r_{0}$ and $z=0.3 r_{0}, z=0.5 r_{0}, z=0.83 r_{0}$, and $z$ $=1.39 r_{0}$, respectively.

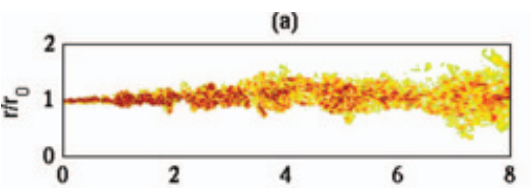

(c)

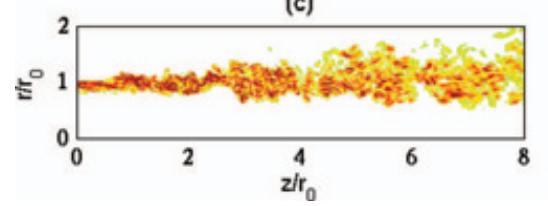

(b)

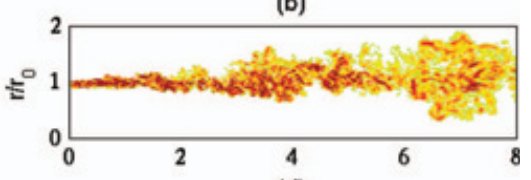

(d)

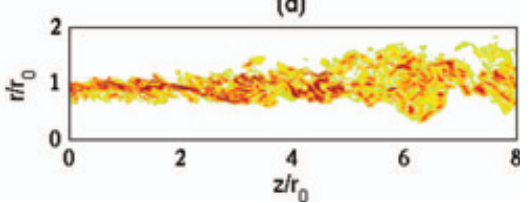

FIG. 19. Snapshots in the $(z, r)$ plane of vorticity norm $|\omega|$ downstream of the nozzle up to $z=8 r_{0}$ for the jets at $\operatorname{Re}_{\theta} \simeq 480$ with (a) $\delta_{0}=0.09 r_{0}$, (b) $\delta_{0}=0.15 r_{0}$, (c) $\delta_{0}=0.25 r_{0}$, (d) $\delta_{0}=0.42 r_{0}$. The color scale ranges up to the level of $17.5 u_{j} / r_{0}, 15.5 u_{j} / r_{0}$, $13.5 u_{j} / r_{0}$, and $11.5 u_{j} / r_{0}$, respectively. Only $r \geq 0$ is shown (enhanced online) [URL: http://dx.doi.org/10.1063/1.4807071.4]. 
(a)

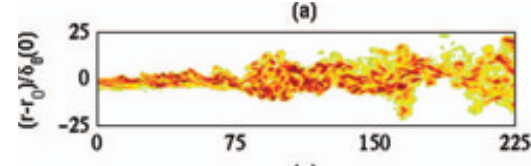

(c)

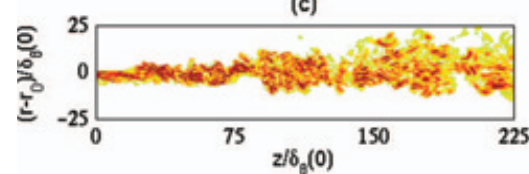

(b)

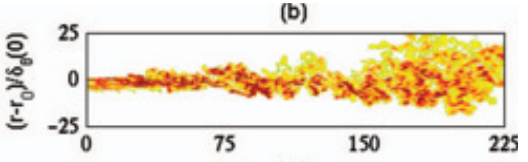

(d)

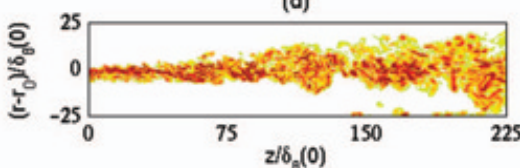

FIG. 20. Snapshots in the $(z, r)$ plane of vorticity norm $|\omega|$, represented using axes normalized by $\delta_{\theta}(0)$, for the jets at $\operatorname{Re}_{\theta}$ $\simeq 480$ with (a) $\delta_{0}=0.09 r_{0}$, (b) $\delta_{0}=0.15 r_{0}$, (c) $\delta_{0}=0.25 r_{0}$, (d) $\delta_{0}=0.42 r_{0}$. The color scale ranges up to the level of $0.42 u_{j} / \delta_{\theta}(0)$ (enhanced online) [URL: http://dx.doi.org/10.1063/1.4807071.5].

Finally, snapshots of the vorticity norm up to $z=20 r_{0}$ in the jets, and of the pressure fluctuations outside, are provided in Figure 21. With increasing nozzle-exit boundary-layer thickness, the jets appear to develop more rapidly, leading to shorter potential cores ending around $z=16 r_{0}$ in Figure 21(a) for $\delta_{0}=0.09 r_{0}$, but only around $z=10 r_{0}$ in Figure $21(\mathrm{~d})$ for $\delta_{0}=0.42 r_{0}$. Concerning the sound pressure fields, strong acoustic waves are emitted in the mixing layers in all cases. They are seen to be of roughly similar amplitudes, but of increasing wavelengths as the value of $\delta_{0} / r_{0}$ grows.

\section{Shear-layer development}

The variations between $z=0$ and $z=10 r_{0}$ of the shear-layer momentum thickness $\delta_{\theta}$ and of the maximum rms values of the axial velocity $u_{z}^{\prime}$ are presented in Figure 22. The profiles of $\delta_{\theta}$

(a)

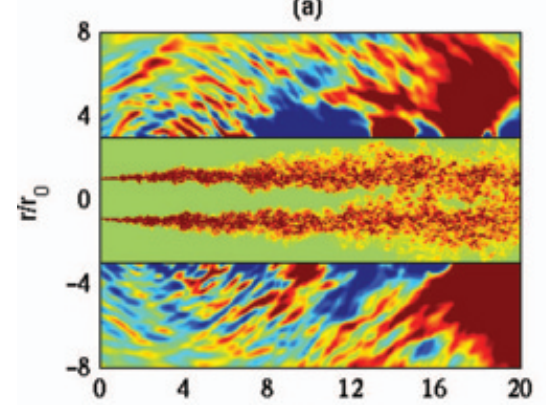

(c)

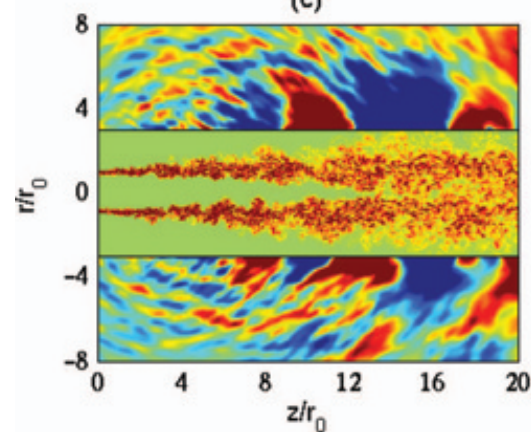

(b)

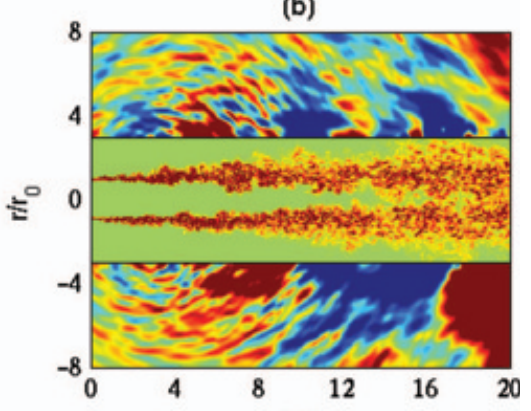

(d)

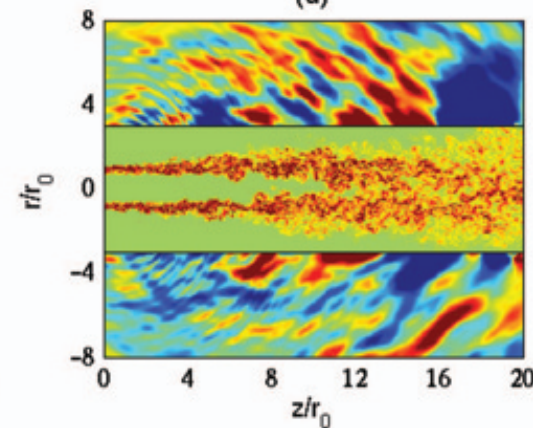

FIG. 21. Snapshots in the $(z, r)$ plane of vorticity norm $|\omega|$ in the flow and of fluctuating pressure $p-p_{a}$ outside, for the jets at $\operatorname{Re}_{\theta} \simeq 480$ with (a) $\delta_{0}=0.09 r_{0}$, (b) $\delta_{0}=0.15 r_{0}$, (c) $\delta_{0}=0.25 r_{0}$, (d) $\delta_{0}=0.42 r_{0}$. The color scales range up to the level of $5.5 u_{j} / r_{0}$ for the vorticity, and from -70 to $70 \mathrm{~Pa}$ for the pressure (enhanced online) [URL: http://dx.doi.org/10.1063/1.4807071.6]. 
(a)

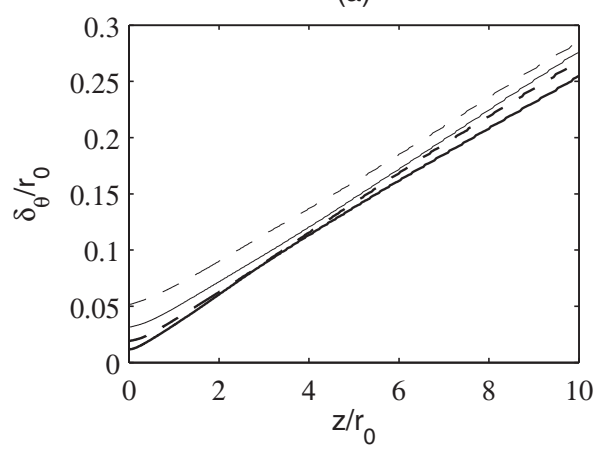

(b)

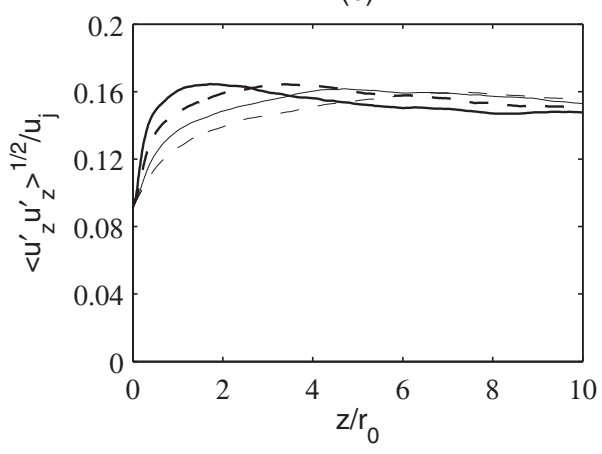

FIG. 22. Variations with $z / r_{0}$ (a) of shear-layer momentum thickness $\delta_{\theta}$ and (b) of peak rms values of fluctuating axial velocity $u_{z}^{\prime}$ for the jets at $\operatorname{Re}_{\theta} \simeq 480$ with (bold solid line) $\delta_{0}=0.09 r_{0}$, (bold dashed line) $\delta_{0}=0.15 r_{0}$, (thin solid line) $\delta_{0}=0.25 r_{0}$, (thin dashed line) $\delta_{0}=0.42 r_{0}$.

do not intersect each other in Figure 22(a), implying that the larger the nozzle-exit boundary-layer thickness, the thicker the mixing layers farther downstream whatever the axial position. The profiles of turbulence intensities also do not appear to differ much in Figure 22(b). The levels of axial velocity fluctuations naturally increase more rapidly with larger $\delta_{0} / r_{0}$, but they reach maximum values, at $z$ $\simeq 1.5 r_{0}$ for $\delta_{0}=0.09 r_{0}, z \simeq 3 r_{0}$ for $\delta_{0}=0.15 r_{0}, z \simeq 4.5 r_{0}$ for $\delta_{0}=0.25 r_{0}$, and $z \simeq 1.5 r_{0}$ for $\delta_{0}$ $=0.42 r_{0}$, which are very close. Peak values between $0.160 u_{j}$ and $0.164 u_{j}$ are indeed found, see in Table VIII for the rms values of radial and azimuthal velocities $u_{r}^{\prime}$ and $u_{\theta}^{\prime}$ and the Reynolds shear stress $\left\langle u_{r}^{\prime} u_{z}^{\prime}\right\rangle$. It can further be emphasized that for all jets the turbulence intensity profiles display a hump, although modest, in the developing mixing layers, which is undoubtedly ${ }^{27}$ due to the low value of $\operatorname{Re}_{\theta}$ in this case.

The variations of $\delta_{\theta} / \delta_{\theta}(0)$ and of the maximum rms values of $u_{z}^{\prime}$ are plotted in Figures 23(a) and 23 (b) as a function of $z / \delta_{\theta}(0)$ up to $z=250 \delta_{\theta}(0)$. The profiles obtained for the four jets are remarkably similar, which were not the case in Figure 9 for the jets at a fixed $\operatorname{Re}_{D}$. The superposition of the curves in Figure 23 shows that the flow transition in the jet mixing layers at Reynolds numbers $\operatorname{Re}_{\theta}$ $\simeq 480$ is independent of the initial shear-layer thickness. It, moreover, suggests grid-convergence of the LES solutions, confirming a posteriori the grid quality, discussed based on a priori arguments in Sec. II C, for all the nozzle-exit boundary layer thicknesses considered in this study.

To verify the very low impact of the nozzle-exit boundary-layer thickness on the features of the shear-layer turbulence, the variations of the integral length scales $L_{u u}^{(z)}$ evaluated from velocity $u_{z}^{\prime}$ along the lip line and the spectra of velocity $u_{r}^{\prime}$ computed at $r=r_{0}$ and $z=10 \delta_{0}$ are represented in Figures 24(a) and 24(b), respectively, using a scaling by $\delta_{\theta}(0)$. The curves obtained for the four jets agree quite well. In particular, the velocity spectra are very similar over the entire Strouhal number range $0 \leq \mathrm{St}_{\theta} \leq 0.06$, that is both around the instability-like component at $\mathrm{St}_{\theta} \simeq 0.012$, whose presence was discussed in Sec. III A 3, refer also to the Appendix, and for high-frequency components. The only notable difference is found for the integral length scales calculated for the jet with $\delta_{0}=0.42 r_{0}$, which are slightly smaller than those for the other jets at $z \geq 150 \delta_{\theta}(0)$. This may be due to the fact that for the jet with $\delta_{0}=0.42 r_{0}, 150 \delta_{\theta}(0) \leq z \leq 250 \delta_{\theta}(0)$ corresponds to $7.7 r_{0}$

TABLE VIII. Peak values of turbulence intensities in the jets at $\operatorname{Re}_{\theta} \simeq 480$.

\begin{tabular}{ccccc}
\hline \hline$\delta_{0} / r_{0}$ & $\left\langle u_{z}^{\prime 2}\right\rangle^{1 / 2} / u_{j}$ & $\left\langle u_{r}^{\prime 2}\right\rangle^{1 / 2} / u_{j}$ & $\left\langle u_{\theta}^{\prime 2}\right\rangle^{1 / 2} / u_{j}$ & $\left\langle u_{r}^{\prime} u_{z}^{\prime}\right\rangle^{1 / 2} / u_{j}$ \\
\hline 0.09 & $16.4 \%$ & $12.7 \%$ & $14.4 \%$ & $10.4 \%$ \\
0.15 & $16.4 \%$ & $12.3 \%$ & $13.9 \%$ & $10.1 \%$ \\
0.25 & $16.2 \%$ & $12.0 \%$ & $13.8 \%$ & $9.9 \%$ \\
0.42 & $16.0 \%$ & $11.6 \%$ & $13.3 \%$ & $9.6 \%$ \\
\hline \hline
\end{tabular}


(a)

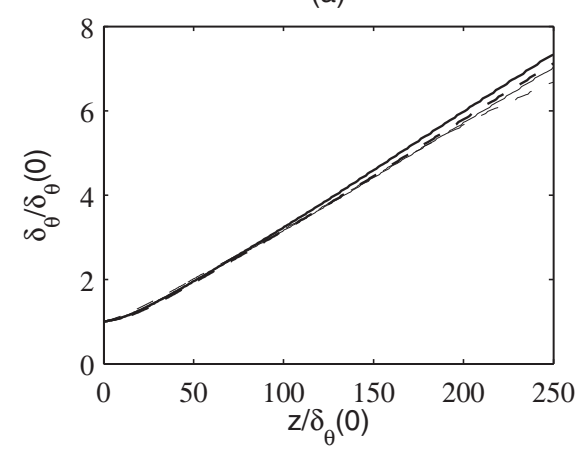

(b)

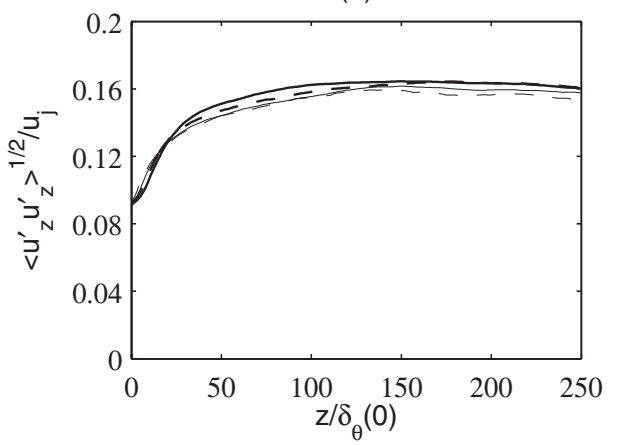

FIG. 23. Variations with $z / \delta_{\theta}(0)$ (a) of shear-layer momentum thickness $\delta_{\theta} / \delta_{\theta}(0)$ and (b) of peak rms values of velocity $u_{z}^{\prime}$ for the jets at $\operatorname{Re}_{\theta} \simeq 480$ with (bold solid line) $\delta_{0}=0.09 r_{0}$, (bold dashed line) $\delta_{0}=0.15 r_{0}$, (thin solid line) $\delta_{0}=0.25 r_{0}$, (thin dashed line) $\delta_{0}=0.42 r_{0}$.

$\leq z \leq 12.9 r_{0}$, which is the flow region where the mixing layers interact and the potential core ends according to Figure 21(d).

\section{Jet development}

The centerline variations of the mean and rms axial velocities obtained for the jets at $\operatorname{Re}_{\theta} \simeq 480$ are presented in Figure 25 together with corresponding measurements ${ }^{4,56,57}$ for Mach number 0.9 jets at $\operatorname{Re}_{D} \geq 5 \times 10^{5}$. In Figure 25(a), larger initial shear-layer thickness leads to shorter potential core lengths decreasing from $z_{c}=16 r_{0}$ for $\delta_{0}=0.09 r_{0}$ down to $z_{c}=11.7 r_{0}$ for $\delta_{0}=0.42 r_{0}$, as reported in Table IX. Downstream of the potential core, the mean axial velocity then seems to decay at a higher rate. Significant differences are also observed between the profiles of axial turbulence intensity in Figure 25(b). In particular, they reach peaks of growing amplitude as the ratio $\delta_{0} / r_{0}$ increases, equal to $11.1 \%$ for $\delta_{0}=0.09 r_{0}, 11.4 \%$ for $\delta_{0}=0.15 r_{0}, 12.5 \%$ for $\delta_{0}=0.25 r_{0}$, and $13.6 \%$ for $\delta_{0}=0.42 r_{0}$. A similar tendency is found for the peak rms values of radial velocity in Table IX. The jets with thicker nozzle-exit boundary layers therefore appear to develop more rapidly with stronger velocity fluctuations on the centerline.

(a)

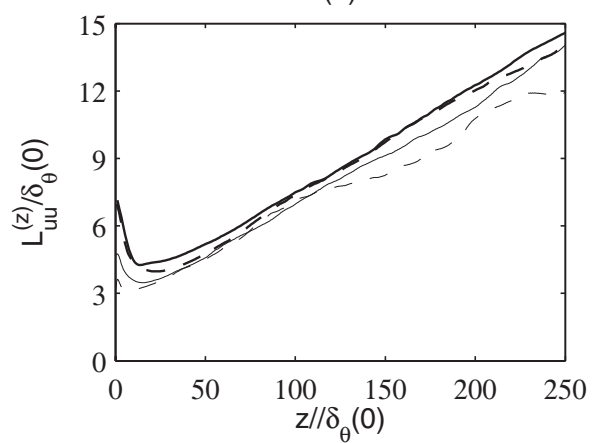

(b)

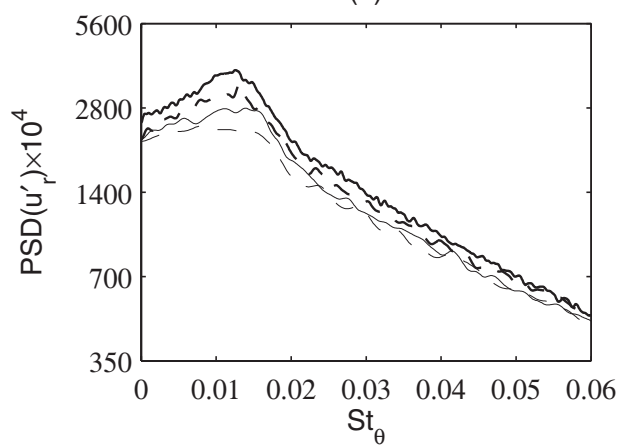

FIG. 24. (a) Variations with $z / \delta_{\theta}(0)$ of integral length scales $L_{u u}^{(z)} / / \delta_{\theta}(0)$ calculated from velocity $u_{z}^{\prime}$ at $r=r_{0}$, and (b) power spectral densities (PSD) normalized by $u_{j}$, as functions of $\mathrm{St}_{\theta}=f \delta_{\theta}(0) / u_{j}$, of velocity $u_{r}^{\prime}$ at $r=r_{0}$ and $z=0.9 r_{0}, z=1.5 r_{0}$, $z=2.5 r_{0}$, and $z=4.17 r_{0}$, respectively, for the jets at $\operatorname{Re}_{\theta} \simeq 480$ with (bold solid line) $\delta_{0}=0.09 r_{0}$, (bold dashed line) $\delta_{0}=0.15 r_{0}$, (thin solid line) $\delta_{0}=0.25 r_{0}$, (thin dashed line) $\delta_{0}=0.42 r_{0}$. 
(a)

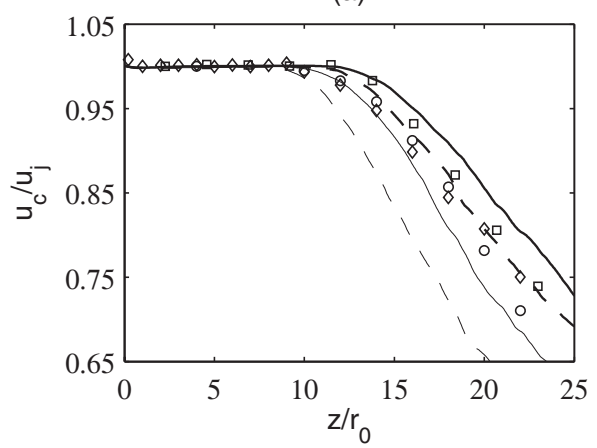

(b)

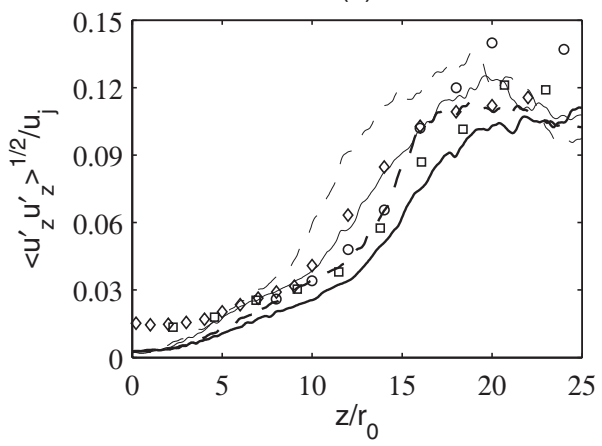

FIG. 25. Variations (a) of centerline mean axial velocity $u_{c}$ and (b) of centerline rms values of velocity $u_{z}^{\prime}$ for the jets at $\operatorname{Re}_{\theta}$ $\simeq 480$ with (bold solid line) $\delta_{0}=0.09 r_{0}$, (bold dashed line) $\delta_{0}=0.15 r_{0}$, (thin solid line) $\delta_{0}=0.25 r_{0}$, (thin dashed line) $\delta_{0}$ $=0.42 r_{0}$. Measurements for jets at $\mathrm{M}=0.9$ and $\operatorname{Re}_{D} \geq 5 \times 10^{5}$ : (circle) Lau et al. ${ }^{56}$ (square) Arakeri et al. ${ }^{4}$ (diamond) Fleury et al. ${ }^{57}$

In support of the findings above, the centerline profiles of mean and rms velocities are shown as a function of $z-z_{c}$ in Figure 26. They clearly illustrate the fact that in the present case thicker initial shear layers result in a faster velocity decay downstream of the potential core and in higher turbulence intensities on the jet axis.

\section{Acoustic fields}

The sound pressure levels calculated at $60 r_{0}$ from the nozzle exit for the jets at $\operatorname{Re}_{\theta} \simeq 480$ are depicted in Figure 27. For radiation angles relative to the flow direction higher than $\phi=50^{\circ}$, the acoustic levels do not differ much depending on the initial shear-layer thickness. In addition, no clear trend is observed. At $\phi=90^{\circ}$, for instance, they vary from $108.2 \mathrm{~dB}$ for $\delta_{0}=0.42 r_{0}$ up to $109.2 \mathrm{~dB}$ for $\delta_{0}=0.15 r_{0}$, as described in Table X. For smaller angles, however, they are found unambiguously to increase with the ratio $\delta_{0} / r_{0}$, thus yielding, at $\phi=40^{\circ}, 115.6 \mathrm{~dB}$ for $\delta_{0}=0.09 r_{0}$ but $117.2 \mathrm{~dB}$ for $\delta_{0}=0.42 r_{0}$, see also in Table X. This result can be related to the strengthening of centerline velocity fluctuations previously noticed for larger $\delta_{0} / r_{0}$. Furthermore, with respect to experimental data $^{58-60}$ for jets at Mach number 0.9 and at diameter-based Reynolds numbers $\operatorname{Re}_{D} \geq 5 \times 10^{5}$, the sound levels are higher by about $3 \mathrm{~dB}$. Given the low Reynolds numbers $\operatorname{Re}_{\theta}$ in the present jets, this is most likely due ${ }^{27}$ to the presence and interactions of large-scale turbulent structures in the mixing layers.

The pressure spectra evaluated at $60 r_{0}$ from the nozzle exit at the angles $\phi=40^{\circ}$ and $90^{\circ}$ are finally shown as a function of Strouhal number $\mathrm{St}_{D}$, and compared to measurements for highReynolds-number jets ${ }^{60,61}$ in Figure 28. As for the jets at $\operatorname{Re}_{D}=5 \times 10^{4}$ in Figure 15, the magnitude

TABLE IX. Axial position of the end of the potential core $z_{c}$, and peak rms values of fluctuating velocities $u_{z}^{\prime}$ and $u_{r}^{\prime}$ on the axis for the jets at $\operatorname{Re}_{\theta} \simeq 480$.

\begin{tabular}{cccc}
\hline \hline$\delta_{0} / r_{0}$ & $z_{c} / r_{0}$ & $\left\langle u_{z}^{\prime 2}\right\rangle^{1 / 2} / u_{j}$ & $\left\langle u_{r}^{\prime 2}\right\rangle^{1 / 2} / u_{j}$ \\
\hline 0.09 & 16.0 & $11.1 \%$ & $8.7 \%$ \\
0.15 & 14.7 & $11.4 \%$ & $9.4 \%$ \\
0.25 & 13.7 & $12.5 \%$ & $9.7 \%$ \\
0.42 & 11.7 & $13.6 \%$ & $10.4 \%$ \\
\hline \hline
\end{tabular}


(a)

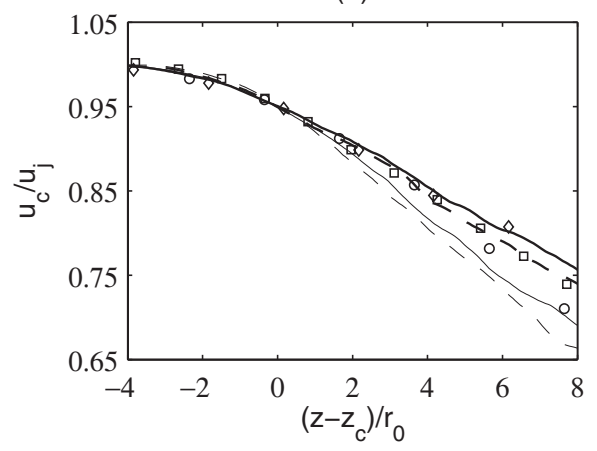

(b)

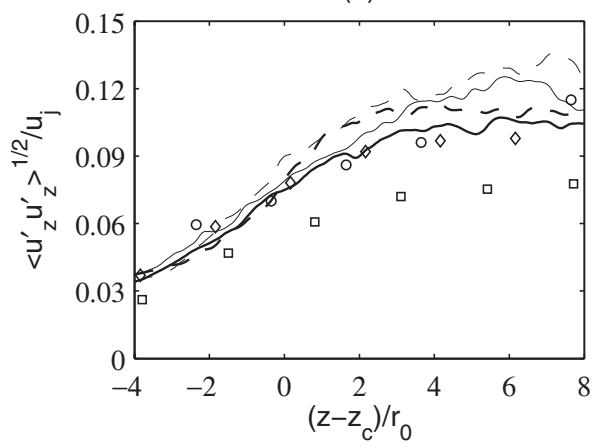

FIG. 26. Variations (a) of centerline mean axial velocity $u_{c}$ and (b) of centerline rms values of velocity $u_{z}^{\prime}$, as a function of $\left(z-z_{c}\right) / r_{0}$, for the jets at $\operatorname{Re}_{\theta} \simeq 480$ with (bold solid line) $\delta_{0}=0.09 r_{0}$, (bold dashed line) $\delta_{0}=0.15 r_{0}$, (thin solid line) $\delta_{0}=0.25 r_{0}$, (thin dashed line) $\delta_{0}=0.42 r_{0}$. Measurements for jets at $\mathrm{M}=0.9$ and $\operatorname{Re}_{D} \geq 5 \times 10^{5}$ : (circle) Lau et al. ${ }^{56}$ (square) Arakeri et al., ${ }^{4}$ (diamond) Fleury et al. ${ }^{57}$

of high-frequency components at $\mathrm{St}_{D} \geq 2$ decreases as the nozzle-exit boundary-layer thickness increases. In this case, however, low-frequency noise does not appear to be reduced. Acoustic components below $\mathrm{St}_{D}=0.8$, on the contrary, become stronger. The change is relatively moderate at $\phi=90^{\circ}$ in Figure 28(b), but is more marked at $\phi=40^{\circ}$ in Figure 28(a). One reason for that may be the higher turbulence intensities obtained at the end of the potential core with rising $\delta_{0} / r_{0}$, leading probably to a stronger noise radiation in the downstream direction. ${ }^{34,37,62-65}$ Another reason may be the shift to lower Strouhal numbers of the frequencies of the waves generated in the mixing layers, which is clearly visible in the pressure fields of Figure 21. Based on previous work, ${ }^{27}$ these noise components are moreover expected to be roughly centered around half the Strouhal number $\mathrm{St}_{\theta}=f \delta_{\theta}(0) / u_{j} \simeq 0.012$ initially dominating in the shear layers according to Figure 24(b), which provides $\mathrm{St}_{D}=1.05,0.63,0.39$, and 0.23 for the jets with $\delta_{0}=0.09 r_{0}, 0.15 r_{0}, 0.25 r_{0}$, and $0.42 r_{0}$, respectively.

\section{SUMMARY AND CONCLUSION}

In this paper, the influence of the nozzle-exit boundary-layer thickness on initially highly disturbed subsonic jets has been investigated for inlet boundary-layer thicknesses $0.09 r_{0} \leq \delta_{0}$ $\leq 0.42 r_{0}$, diameter Reynolds numbers $1.8 \times 10^{4} \leq \mathrm{Re}_{D} \leq 8.3 \times 10^{4}$ and momentum-thickness Reynolds numbers $304 \leq \operatorname{Re}_{\theta} \leq 1288$. Jets at a constant $\operatorname{Re}_{D}=5 \times 10^{4}$ as well as jets at nearly identical $\operatorname{Re}_{\theta} \simeq 480$ have been considered, in order to distinguish between the effects of $\delta_{0} / r_{0}$ and $\operatorname{Re}_{\theta}$. In that sense, this work is a continuation of and a complement to a recent study ${ }^{27}$ dealing with the sensitivity to Reynolds number of tripped jets with a fixed $\delta_{0}=0.15 r_{0}$ but at Reynolds numbers $2.5 \times 10^{4} \leq \operatorname{Re}_{D} \leq 2 \times 10^{5}$, and consequently $256 \leq \operatorname{Re}_{\theta} \leq 1856$.

Some key results obtained in the mixing layers, in the jets and in the acoustic fields are represented as a function of $\delta_{0} / r_{0}$ in Figures 29-31 using solid lines for the cases at $\operatorname{Re}_{D}=5 \times 10^{4}$

TABLE X. Overall sound pressure levels at $60 r_{0}$ from the nozzle exit at $\phi$ $=40^{\circ}$ and $\phi=90^{\circ}$ for the jets at $\operatorname{Re}_{\theta} \simeq 480$.

\begin{tabular}{lll}
\hline \hline$\delta_{0} / r_{0}$ & $\phi=40^{\circ}$ & $\phi=90^{\circ}$ \\
\hline 0.09 & $115.6 \mathrm{~dB}$ & $108.7 \mathrm{~dB}$ \\
0.15 & $116.6 \mathrm{~dB}$ & $109.2 \mathrm{~dB}$ \\
0.25 & $116.8 \mathrm{~dB}$ & $108.9 \mathrm{~dB}$ \\
0.42 & $117.2 \mathrm{~dB}$ & $108.2 \mathrm{~dB}$ \\
\hline \hline
\end{tabular}




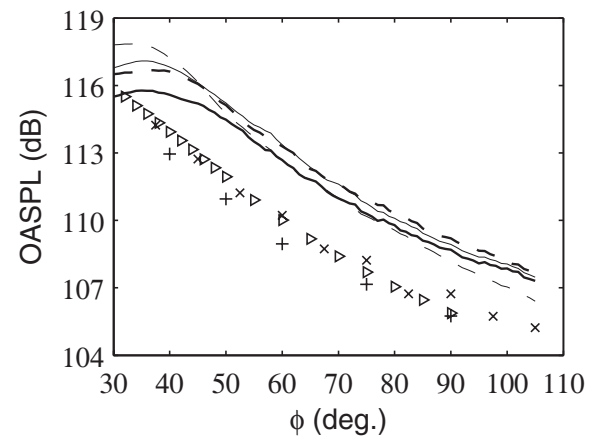

FIG. 27. Overall sound pressure levels (OASPL) at $60 r_{0}$ from the nozzle exit, as a function of the angle $\phi$, for the jets at $\operatorname{Re}_{\theta} \simeq 480$ with (bold solid line) $\delta_{0}=0.09 r_{0}$, (bold dashed line) $\delta_{0}=0.15 r_{0}$, (thin solid line) $\delta_{0}=0.25 r_{0}$, (thin dashed line) $\delta_{0}=0.42 r_{0}$. Measurements for jets at $\operatorname{Re}_{D} \geq 5 \times 10^{5}$ : (plus) Mollo-Christensen et al., ${ }^{58}$ (times) Lush, ${ }^{59}$ (right-pointing triangle) Bogey et al. ${ }^{60}$

(a)

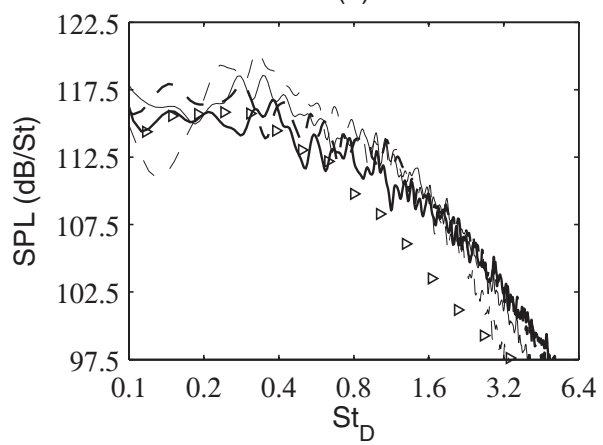

(b)

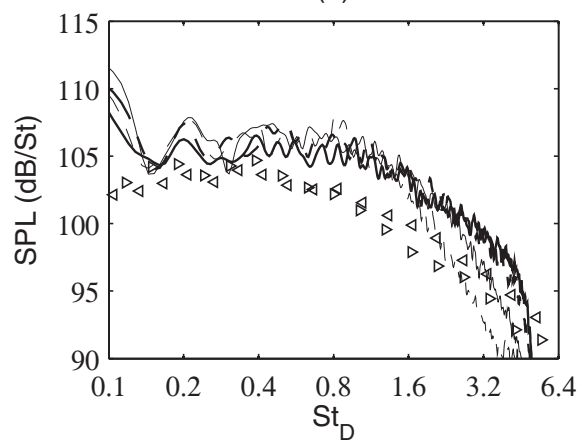

FIG. 28. Sound pressure levels (SPL) at $60 r_{0}$ from the nozzle exit, as a function of $\mathrm{St}_{D}$, at the angles $\phi$ of (a) $40^{\circ}$ and (b) $90^{\circ}$ for the jets at $\operatorname{Re}_{\theta} \simeq 480$ with (bold solid line) $\delta_{0}=0.09 r_{0}$, (bold dashed line) $\delta_{0}=0.15 r_{0}$, (thin solid line) $\delta_{0}=0.25 r_{0}$, (thin dashed line) $\delta_{0}=0.42 r_{0}$. Measurements for jets at $\operatorname{Re}_{D} \geq 7.8 \times 10^{5}$ : (left-pointing triangle) Tanna, ${ }^{61}$ (right-pointing triangle) Bogey et al. . $^{60}$

(a)

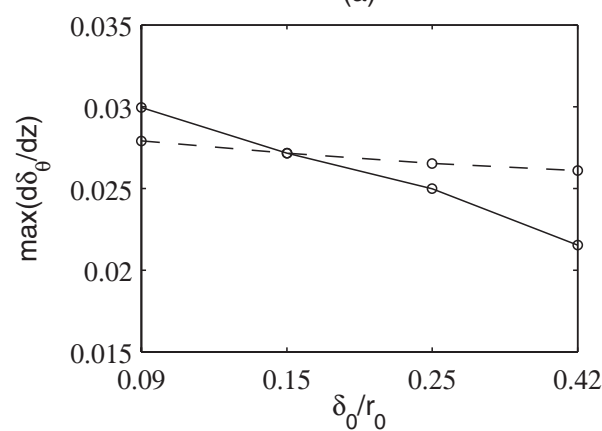

(b)

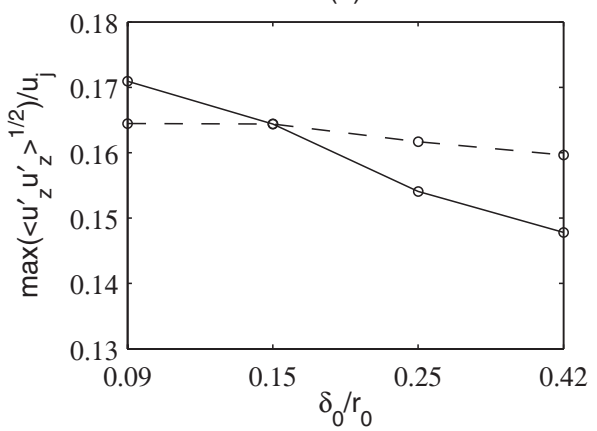

FIG. 29. Variations with $\delta_{0} / r_{0}$ (a) of the maximum value of the shear-layer spreading rate $\mathrm{d} \delta_{\theta} / \mathrm{d} z$, and (b) of the peak rms value of velocity $u_{z}^{\prime}$ in the entire jets, for (solid line) $\operatorname{Re}_{D}=5 \times 10^{4}$ and (dashed line) $\operatorname{Re}_{\theta} \simeq 480$. 
(a)

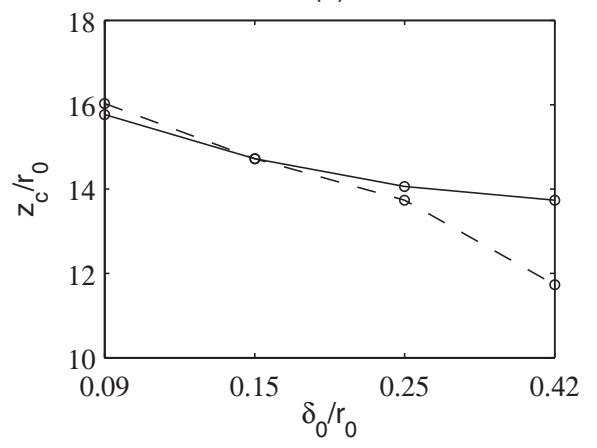

(b)

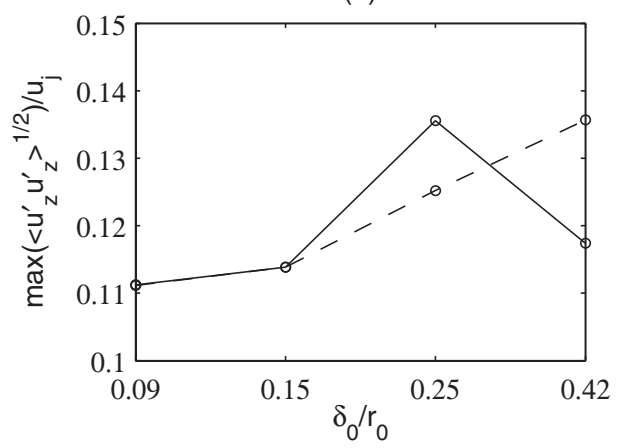

FIG. 30. Variations with $\delta_{0} / r_{0}$ (a) of the axial position of the end of the potential core $z_{c}$, and (b) of the peak rms value of velocity $u_{z}^{\prime}$ on the jet axis, for (solid line) $\operatorname{Re}_{D}=5 \times 10^{4}$ and (dashed line) $\operatorname{Re}_{\theta} \simeq 480$.

and dashed lines for those at $\operatorname{Re}_{\theta} \simeq 480$. In Figure 29, increasing the nozzle-exit boundary-layer thickness appears to have a strong influence on the mixing-layer properties for the jets at $\operatorname{Re}_{D}=5 \times$ $10^{4}$, but to have a limited impact for those at $\operatorname{Re}_{\theta} \simeq 480$. More precisely, shear-layer spreading rates and turbulence intensities are lower as the ratio $\delta_{0} / r_{0}$, and $\mathrm{Re}_{\theta}$, grow in the former case, whereas they do not vary much in the latter case. The flow development just downstream of the nozzle exit thus essentially depends on $\operatorname{Re}_{\theta}$, that is on viscosity effects.

In Figure 30, contrary to previous figure, the main jet flow characteristics are found to be more sensitive to $\delta_{0} / r_{0}$ at $\operatorname{Re}_{\theta} \simeq 480$ than at $\operatorname{Re}_{D}=5 \times 10^{4}$. For the jets with a nearly identical $\operatorname{Re}_{\theta}$, it is indeed clearly observed that thicker exit boundary layers result in a shorter potential core and higher centerline velocity fluctuations, whereas tendencies are less evident for the other jets. This can be explained by the fact that at a constant Reynolds number $\mathrm{Re}_{D}$, the variations of the jet properties with $\delta_{0}$ result from the modifications of both $\delta_{0} / r_{0}$ and $\operatorname{Re}_{\theta}$. Consequently, geometry effects, such as, for instance, the shortening of the jet potential core with increasing $\delta_{0} / r_{0}$, may be countered by viscosity effects in the mixing layers, such as the reduction of their spreading rate with $\operatorname{Re}_{\theta}$.

Concerning the far-field pressure levels, they are noted in Figure 31 to depend appreciably on the initial shear-layer thickness $\delta_{0}$, as expected given the changes in turbulence intensities reported above. Increasing $\delta_{0}$ for a fixed jet diameter generally leads to weaker sound levels at all radiation angles because of the corresponding growth of the Reynolds number $\operatorname{Re}_{\theta}$. In the case where $\operatorname{Re}_{\theta}$ remains the same, however, rising $\delta_{0} / r_{0}$ has a smaller impact on noise levels, except for a strengthening in the downstream direction.

(a)

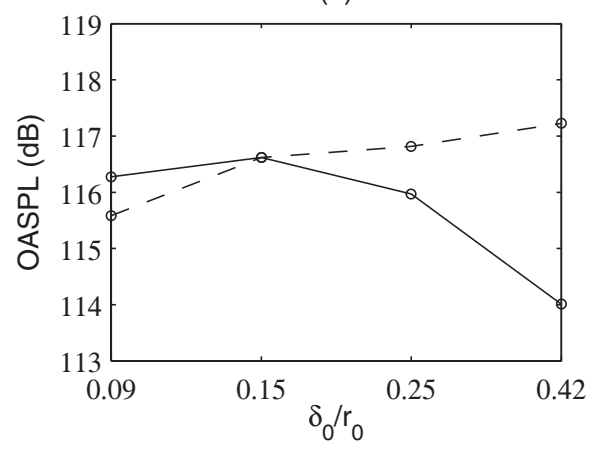

(b)

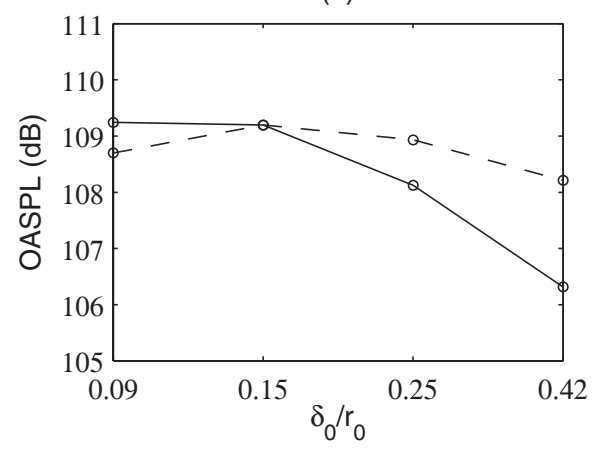

FIG. 31. Variations with $\delta_{0} / r_{0}$ of the overall sound pressure levels (OASPL) at $60 r_{0}$ from the pipe exit, at the angles $\phi$ of (a) $40^{\circ}$ and (b) $90^{\circ}$, for the jets at (solid line) $\operatorname{Re}_{D}=5 \times 10^{4}$ and (dashed line) $\operatorname{Re}_{\theta} \simeq 480$. 
To briefly conclude, this work demonstrates the importance of the initial shear-layer thickness in subsonic jets with disturbed upstream flow conditions, in terms of both geometry and viscosity effects, induced by the variations of the ratio $\delta_{0} / r_{0}$ and of the momentum-thickness Reynolds number $\operatorname{Re}_{\theta}$, respectively. These effects can reinforce or counteract each other, which might render difficult any a priori prediction on flow and sound fields. This is in particular the case in recent jet experiments ${ }^{8,12}$ using ASME and conic nozzles, in which the exit flow conditions have been well documented. Viscosity effects can, nevertheless, be expected to dominate in jets at low and moderate Reynolds numbers, but to be rather weak in jets at high Reynolds numbers, typically above a threshold value of about $\operatorname{Re}_{\theta}=1000$, which should be the case in full-scale industrial jets, but not necessarily in model-scale university jets.

\section{ACKNOWLEDGMENTS}

This work was granted access to the HPC resources of the Institut du Développement et des Ressources en Informatique Scientifique (IDRIS) under the allocation 2011-020204 made by GENCI (Grand Equipement National de Calcul Intensif).

\section{APPENDIX: AMPLIFICATION RATES OF LINEAR INSTABILITY WAVES DOWNSTREAM OF THE JET NOZZLE EXIT}

In this appendix, the amplification rates of instability waves in axisymmetric mixing layers representative of the mean flows obtained downstream of the jet nozzle exit in the present study are examined based on a linear spatial stability analysis. Following Michalke, ${ }^{19}$ hyperbolic-tangent axisymmetric velocity profiles $u_{z}(r) / u_{j}=\left[1+\tanh \left(\left(r_{0}-r\right) /\left(2 \delta_{\theta}\right)\right)\right] / 2$ are chosen, where $r_{0}$ is the radius and $\delta_{\theta}$ is the momentum thickness of the mixing layers. The values of $\delta_{\theta}$ are set to $0.012 r_{0}$, $0.019 r_{0}, 0.031 r_{0}$, and $0.051 r_{0}$, in order to match the nozzle-exit conditions reported in Tables III and VII for the jets with inlet boundary-layer thickness $\delta_{0}=0.09 r_{0}, 0.15 r_{0}, 0.25 r_{0}$, and $0.42 r_{0}$. The velocity $u_{j}$ is also fixed to provide a Mach number $\mathrm{M}=u_{j} / c_{0}$ equal to 0.9 . A generalized Rayleigh equation is consequently solved to include the compressibility effects in the stability analysis. ${ }^{22}$ Viscous effects are not taken into account because they are expected to be very small at the Reynolds numbers $\operatorname{Re}_{\theta} \geq 304$ considered in this paper according to the work by Morris. ${ }^{21}$

The dimensionless growth rates $-\operatorname{Im}\left(k_{z}\right) \delta_{\theta}$, where $\operatorname{Im}\left(k_{z}\right)$ is the imaginary part of the axial wave number $k_{z}$, are calculated for the first two azimuthal modes $n_{\theta}=0$ and 1 , which are the most unstable modes. They are plotted in Figures 32(a) and 32(b) as a function of Strouhal number $\mathrm{St}_{\theta}=f \delta_{\theta} / u_{j}$. In good agreement with the results presented in the review of Michalke, ${ }^{19}$ the amplification curves obtained for $\delta_{\theta} \leq 0.031 r_{0}$ are very similar. For $\delta_{\theta}=0.051 r_{0}$, compared to the first three cases, a

(a)

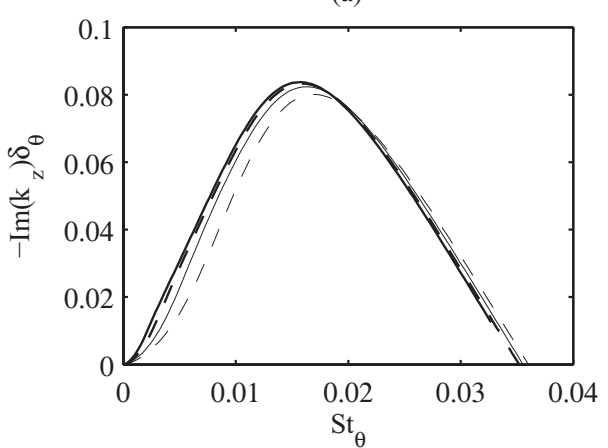

(b)

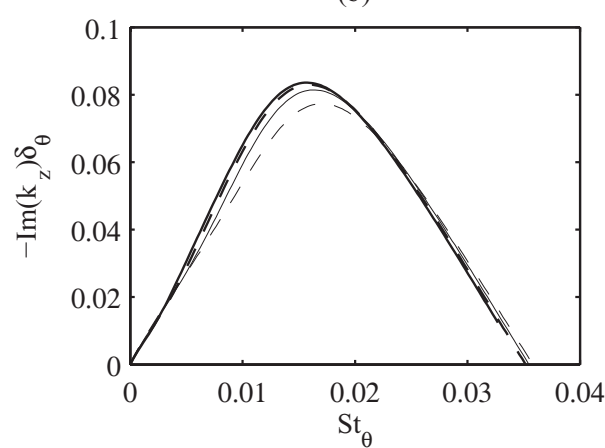

FIG. 32. Amplification rates $-\operatorname{Im}\left(k_{z}\right) \delta_{\theta}$ as a function of Strouhal number $\mathrm{St}_{\theta}=f \delta_{\theta} / u_{j}$ of linear instability waves for azimuthal modes (a) $n_{\theta}=0$, and (b) $n_{\theta}=1 \mathrm{in}$ an hyperbolic-tangent axisymmetric mixing layer at $\mathrm{M}=0.9$ with: (bold solid line) $\delta_{\theta}$ $=0.012 r_{0}$, (bold dashed line) $\delta_{\theta}=0.019 r_{0}$, (thin solid line) $\delta_{\theta}=0.031 r_{0}$, (thin dashed line) $\delta_{\theta}=0.051 r_{0}$. 
slight decrease of the growth rates is observed for lower frequencies for the axisymmetric mode, and for $0.005 \leq \mathrm{St}_{\theta} \leq 0.02$ for mode $n_{\theta}=1$. In all cases and for both modes, however, maximum growth rates $-\operatorname{Im}\left(k_{z}\right) \delta_{\theta} \simeq 0.08$ are reached around $\mathrm{St}_{\theta}=0.016$.

${ }^{1}$ A. K. M. F. Hussain, "Coherent structures-reality and myth,” Phys. Fluids 26(10), 2816 (1983)

${ }^{2}$ D. G. Crighton, “Acoustics as a branch of fluid mechanics," J. Fluid Mech. 106, 261 (1981).

${ }^{3}$ P. J. Morris and K. B. M. Q. Zaman, "Velocity measurements in jets with application to noise source modelling," J. Sound Vib. 329(4), 394 (2010).

${ }^{4}$ V. H. Arakeri, A. Krothapalli, V. Siddavaram, M. B. Alkislar, and L. Lourenco, "On the use of microjets to suppress turbulence in a Mach 0.9 axisymmetric jet," J. Fluid Mech. 490, 75 (2003).

${ }^{5}$ S. C. Crow and F. H. Champagne, "Orderly structure in jet turbulence," J. Fluid Mech. 48, 547 (1971).

${ }^{6}$ K. B. M. Q. Zaman, "Effect of the initial condition on subsonic jet noise," AIAA J. 23(9), 1370 (1985).

${ }^{7}$ K. B. M. Q. Zaman, "Far-field noise of a subsonic jet under controlled excitation," J. Fluid Mech. 152, 83 (1985).

${ }^{8}$ K. B. M. Q. Zaman, "Effect of initial boundary-layer state on subsonic jet noise," AIAA J. 50(8), 1784 (2012).

${ }^{9}$ R. C. Deo, J. Mi, and G. J. Nathan, “The influence of Reynolds number on a plane jet," Phys. Fluids 20(1), 075108 (2008).

${ }^{10}$ H. A. Becker and T. A. Massaro, "Vortex evolution in a round jet," J. Fluid Mech. 31(3), 435 (1968).

${ }^{11}$ K. K. Ahuja, "An experimental study of subsonic jet noise with particular reference to the effects of upstream disturbances," M.S. thesis (University of London, 1972).

${ }^{12}$ A. Z. Karon and K. K. Ahuja, "Effect of nozzle-exit boundary layer on jet noise," AIAA Paper 2013-0615, 2013.

${ }^{13}$ M. A. Z. Hasan and A. K. M. F. Hussain, "The self-excited axisymmetric jet," J. Fluid Mech. 115, 59 (1982).

${ }^{14}$ K. Viswanathan and L. T. Clark, "Effect of nozzle internal contour on jet aeroacoustics," Int. J. Aeroacoust. 3(2), 103 (2004).

${ }^{15}$ M. Harper-Bourne, “Jet noise measurements: past and present,” Int. J. Aeroacoust. 9(4\&5), 559 (2010).

${ }^{16}$ J. E. Bridges and A. K. M. F. Hussain, "Roles of initial conditions and vortex pairing in jet noise," J. Sound Vib. 117(2), 289 (1987).

${ }^{17}$ G. Raman, K. B. M. Q. Zaman, and E. J. Rice, "Initial turbulence effect on jet evolution with and without tonal excitation," Phys. Fluids A 1(7), 1240 (1989).

${ }^{18}$ A. K. M. F. Hussain and M. F. Zedan, "Effects of the initial condition on the axisymmetric free shear layer: Effects of the initial momentum thickness," Phys. Fluids 21(7), 1100 (1978).

${ }^{19}$ A. Michalke, "Survey on jet instability theory," Prog. Aerosp. Sci. 21, 159 (1984).

${ }^{20} \mathrm{~J}$. Cohen and I. Wygnanski, "The evolution of instabilities in the axisymmetric jet. Part 1. The linear growth of disturbances near the nozzle,” J. Fluid Mech. 176, 191 (1987).

${ }^{21}$ P. J. Morris, "The spatial viscous instability of axisymmetric jets," J. Fluid Mech. 77(3), 511 (1976).

${ }^{22}$ P. J. Morris, "Viscous stability of compressible axisymmetric jets," AIAA J. 21(4), 481 (1983).

${ }^{23}$ E. Gutmark and C.-M. Ho, "Preferred modes and the spreading rates of jets," Phys. Fluids 26(10), 2932 (1983).

${ }^{24} \mathrm{~K}$. Gudmundsson and T. Colonius, "Instability wave models for the near-field fluctuations of turbulent jets," J. Fluid Mech. 689, 97 (2011).

${ }^{25}$ G. L. Brown and A. Roshko, "Density effect and large structure in turbulent mixing layers," J. Fluid Mech. 64, 775 (1974).

${ }^{26}$ R. R. Kleinman and J. B. Freund, "The sound from mixing layers simulated with different ranges of turbulent scales," Phys. Fluids 20(10), 101503 (2008).

${ }^{27}$ C. Bogey, O. Marsden, and C. Bailly, "Effects of moderate Reynolds numbers on subsonic round jets with highly disturbed nozzle-exit boundary layers," Phys. Fluids 24(10), 105107 (2012).

${ }^{28}$ T. Colonius and S. K. Lele, "Computational aeroacoustics: Progress on nonlinear problems of sound generation," Prog. Aerosp. Sci. 40, 345 (2004).

${ }^{29}$ C. Bailly and C. Bogey, "Contributions of CAA to jet noise research and prediction," Int. J. Comput. Fluid Dyn. 18(6), 481 (2004)

${ }^{30}$ M. Wang, J. B. Freund, and S. K. Lele, "Computational prediction of flow-generated sound," Annu. Rev. Fluid Mech. 38, 483 (2006).

${ }^{31}$ S. A. Stanley and S. Sarkar, "Influence of nozzle conditions and discrete forcing on turbulent planar jets," AIAA J. 38(9), 1615 (2000)

${ }^{32}$ C. Bogey and C. Bailly, "Effects of inflow conditions and forcing on a Mach 0.9 jet and its radiated noise," AIAA J. 43(5), 1000 (2005).

${ }^{33}$ J. Kim and H. Choi, "Large-eddy simulation of a circular jet: Effect of inflow conditions on the near field," J. Fluid Mech. 620, 383 (2009).

${ }^{34} \mathrm{C}$. Bogey and C. Bailly, "Influence of nozzle-exit boundary-layer conditions on the flow and acoustic fields of initially laminar jets," J. Fluid Mech. 663, 507 (2010).

${ }^{35} \mathrm{C}$. Bogey, O. Marsden, and C. Bailly, "Large-eddy simulation of the flow and acoustic fields of a Reynolds number $10^{5}$ subsonic jet with tripped exit boundary layers," Phys. Fluids 23(3), 035104 (2011).

${ }^{36} \mathrm{C}$. Bogey, O. Marsden, and C. Bailly, "On the spectra of nozzle-exit velocity disturbances in initially nominally turbulent jets,” Phys. Fluids 23(9), 091702 (2011).

${ }^{37} \mathrm{C}$. Bogey, O. Marsden, and C. Bailly, "Influence of initial turbulence level on the flow and sound fields of a subsonic jet at a diameter-based Reynolds number of $10^{5}$," J. Fluid Mech. 701, 352 (2012).

${ }^{38}$ K. Mohseni and T. Colonius, "Numerical treatment of polar coordinate singularities," J. Comput. Phys. 157(2), 787 (2000).

${ }^{39} \mathrm{C}$. Bogey, N. de Cacqueray, and C. Bailly, "Finite differences for coarse azimuthal discretization and for reduction of effective resolution near origin of cylindrical flow equations," J. Comput. Phys. 230(4), 1134 (2011).

${ }^{40}$ C. Bogey and C. Bailly, "A family of low dispersive and low dissipative explicit schemes for flow and noise computations," J. Comput. Phys. 194(1), 194 (2004). 
${ }^{41}$ C. Bogey, N. de Cacqueray, and C. Bailly, "A shock-capturing methodology based on adaptative spatial filtering for high-order nonlinear computations,” J. Comput. Phys. 228(5), 1447 (2009).

${ }^{42}$ J. Berland, C. Bogey, O. Marsden, and C. Bailly, "High-order, low dispersive and low dissipative explicit schemes for multiple-scale and boundary problems," J. Comput. Phys. 224(2), 637 (2007).

${ }^{43}$ C. K. W. Tam and Z. Dong, "Radiation and outflow boundary conditions for direct computation of acoustic and flow disturbances in a nonuniform mean flow," J. Comput. Acoust. 4(2), 175 (1996).

${ }^{44} \mathrm{C}$. Bogey and C. Bailly, "Three-dimensional non reflective boundary conditions for acoustic simulations: far-field formulation and validation test cases," Acta Acust. United Ac. 88(4), 463 (2002).

${ }^{45}$ C. Bogey and C. Bailly, "Large-eddy simulations of transitional round jets: Influence of the Reynolds number on flow development and energy dissipation,” Phys. Fluids 18(6), 065101 (2006).

${ }^{46}$ C. Bogey and C. Bailly, "Large-eddy simulations of round free jets using explicit filtering with/without dynamic Smagorinsky model,” Int. J. Heat Fluid Flow 27(4), 603 (2006).

${ }^{47} \mathrm{C}$. Bogey and C. Bailly, "Turbulence and energy budget in a self-preserving round jet: Direct evaluation using large-eddy simulation," J. Fluid Mech. 627, 129 (2009).

${ }^{48}$ D. Fauconnier, C. Bogey, and E. Dick, “On the performance of relaxation filtering for large-eddy simulation,” J. Turbul. 14(1), 22 (2013).

${ }^{49}$ C. Bogey, S. Barré, D. Juvé, and C. Bailly, "Simulation of a hot coaxial jet: Direct noise prediction and flow-acoustics correlations," Phys. Fluids 21(3), 035105 (2009).

${ }^{50}$ K. K. Ahuja, B. J. Tester, and H. K. Tanna, "Calculation of far field jet noise spectra from near field measurements with true source location,” J. Sound Vib. 116(3), 415 (1987).

${ }^{51}$ R. G. Batt, "Some measurements on the effect of tripping the two-dimensional shear layer," AIAA J. 13(2), 245 (1975).

52 J. G. M. Eggels, F. Unger, M. H. Weiss, J. Westerweel, R. J. Adrian, R. Friedrich, and F. T. M. Nieuwstadt, "Fully developed turbulent pipe flow: A comparison between direct numerical simulation and experiment," J. Fluid Mech. 268, 175 (1994).

${ }^{53}$ C. D. Tomkins and R. J. Adrian, "Energetic spanwise modes in the logarithmic layer of a turbulent boundary layer," J. Fluid Mech. 545, 141 (2005).

${ }^{54}$ P. O. A. L. Davies, M. J. Fisher, and M. J. Barratt, "The characteristics of the turbulence in the mixing region of a round jet," J. Fluid Mech. 15, 337 (1963).

${ }^{55}$ B. G. Jones, H. P. Planchon, and R. J. Hammersley, "Turbulent correlation measurements in a two-stream mixing layer," AIAA J. 11(8), 1146 (1973).

${ }^{56}$ J. C. Lau, P. J. Morris, and M. J. Fisher, "Measurements in subsonic and supersonic free jets using a laser velocimeter," J. Fluid Mech. 93(1), 1 (1979).

${ }^{57}$ V. Fleury, C. Bailly, E. Jondeau, M. Michard, and D. Juvé, "Space-time correlations in two subsonic jets using dual-PIV measurements," AIAA J. 46(10), 2498 (2008)

${ }^{58}$ E. Mollo-Christensen, M. A. Kolpin, and J. R. Martucelli, "Experiments on jet flows and jet noise far-field spectra and directivity patterns," J. Fluid Mech. 18, 285 (1964).

${ }^{59}$ P. A. Lush, "Measurements of subsonic jet noise and comparison with theory," J. Fluid Mech. 46(3), 477 (1971).

${ }^{60}$ C. Bogey, S. Barré, V. Fleury, C. Bailly, and D. Juvé, "Experimental study of the spectral properties of near-field and far-field jet noise," Int. J. Aeroacoust. 6(2), 73 (2007).

${ }^{61}$ H. K. Tanna, "An experimental study of jet noise. Part I: Turbulent mixing noise," J. Sound Vib. 50(3), 405 (1977).

${ }^{62}$ C. Bogey, C. Bailly, and D. Juvé, "Noise investigation of a high subsonic, moderate Reynolds number jet using a compressible LES,” Theor. Comput. Fluid Dyn. 16(4), 273 (2003).

${ }^{63}$ J. Panda, R. G. Seasholtz, and K. A. Elam, "Investigation of noise sources in high-speed jets via correlation measurements," J. Fluid Mech. 537, 349 (2005).

${ }^{64} \mathrm{C}$. Bogey and C. Bailly, "An analysis of the correlations between the turbulent flow and the sound pressure field of subsonic jets,” J. Fluid Mech. 583, 71 (2007).

${ }^{65}$ C. K. W. Tam, K. Viswanathan, K. K. Ahuja, and J. Panda, "The sources of jet noise: Experimental evidence," J. Fluid Mech. 615, 253 (2008) 